Pacific Journal of Mathematics

RELATIVE DIMENSION, TOWERS OF PROJECTIONS AND
COMMUTING SQUARES OF SUBFACTORS 


\title{
RELATIVE DIMENSION, TOWERS OF PROJECTIONS AND COMMUTING SQUARES OF SUBFACTORS
}

\author{
SORIN POPA \\ Dedicated to the memory of Henry Dye
}

\begin{abstract}
We study the set of projections of the type $\mathrm{II}_{1}$ factor $M$ which expected on the subfactor $N \subset M$ are scalar multiples of the identity. The set of all these scalars, denoted $\Lambda(M, N)$, is an invariant for the inclusion $N \subset M$. We compute $\Lambda(M, N)$ when $[M: N]<4$, when $N$ is locally trivial and some parts of $\Lambda(M, N)$ when $[M: N]>4$. We prove that projections expected on the same scalar in $N$ are conjugate by a unitary element in $N$. We apply all these to the commuting square problem.
\end{abstract}

Introduction. In a type $\mathrm{II}_{1}$ factor a projection may have any dimension between 0 and 1 . This corresponds to the fact that Hilbert modules $H$ over a type $\mathrm{II}_{1}$ factor $M$ may have any positive number as (relative) dimension $\operatorname{dim}_{M} H$ [9].

There has been more and more evidence in the past ten years or so that it is much more useful to regard a type $\mathrm{II}_{1}$ factor $M$ together with its subalgebras $N$ and more generally to consider pairs of arbitrary algebras $M, N$. The corresponding appropriate notion of module is then the one introduced by Connes in [2], the $N-M$ Hilbert bimodules (or correspondences).

If $N \subset M$ is a subfactor of the type $\mathrm{II}_{1}$ factor $M$ then $\mathrm{V}$. Jones had the idea to consider the number

$$
\operatorname{dim}_{N} H / \operatorname{dim}_{M} H\left(=\operatorname{dim}_{N} H \operatorname{dim}_{M^{\prime}} H\right)
$$

as an invariant up the conjugacy by automorphisms of $M$ for the subfactor $N$ (this number is independent of $H$ by [9]). Jones called this number the index of $N$ in $M$ denoting it [ $M: N]$. One of his remarkable results in [6] is that $[M: N]$ can only take the values $\left\{4 \cos ^{2} \pi /(n+2) \mid n \geq 0\right\} \cup[4, \infty]$.

The number $[M: N]$ can also be interpreted in a more intrinsic way: it is the dimension of the smallest nonzero projection in $M$ which expected on $N$ is a scalar multiple of the identity (by [6] and [12]). This is somehow related to the fact that $[M: N]$ can be viewed as the minimal possible dimension $\operatorname{dim}_{M, N} H \stackrel{\text { def }}{=} \operatorname{dim}_{M} H \operatorname{dim}_{N} H([14])$ 
of an $N-M$ Hilbert bimodule which is the restriction of an $M-M$ bimodule.

The conditional expectation $E_{N}(e)$ of a projection $e \in M$ on the subfactor $N$ may be regarded as the dimension of the projection $e$ relative to the subfactor $N$. The interesting case is when $E_{N}(e)$ is a scalar multiple of the identity. In this paper we begin the study of the geometry of such projections. The set $\Lambda(M, N)$ of nonzero scalars appearing this way is of course an invariant for $N \subset M$. This invariant is in fact closely related to the index $[M: N]$. Our results here deal with the description of $\Lambda(M, N)$ and with the conjugacy problem by unitary elements in $N$ of the projections which have the same (scalar) relative dimension over $N$.

To state our main result denote by $P_{n}(x)$ the Jones' polynomials defined recursively by $P_{-1} \equiv 1, P_{0} \equiv 1, P_{n+1}(x)=P_{n}(x)-x P_{n-1}(x), n>$ 0 . By [6] these polynomials have the property that

$$
P_{k}\left(\left(4 \cos ^{2} \pi /(n+2)\right)^{-1}\right)>0, \quad 0 \leq k \leq n-1,
$$

and $P_{n}\left(\left(4 \cos ^{2} \pi /(n+2)\right)^{-1}\right)=0$. Moreover $P_{k}(\varepsilon)>0$ for all $k \geq 0$ and $\varepsilon \leq 1 / 4$.

THEOREM. Let $N \subset M$ be a subfactor of finite index.

(i) If $[M: N]=4 \cos ^{2} \pi /(n+2)$ for some $n \geq 1$ then $\Lambda(M, N)=$ $\{0\} \cup\left\{P_{k-1}\left([M: N]^{-1}\right) /[M: N] P_{k}\left([M: N]^{-1}\right) \mid 0 \leq k \leq n-1\right\}$

(ii) If $[M: N] \geq 4$ and $t \leq 1 / 2$ is so that $t(1-t)=[M: N]^{-1}$ then $\Lambda(M, N) \cap(0, t)=\left\{P_{k-1}\left([M: N]^{-1}\right) /[M: N] P_{k}\left([M: N]^{-1}\right) \mid k \geq 0\right\}$.

Moreover, if $[M: N]<4$ (respectively $[M: N] \geq 4$ ) and $f_{1}, f_{2}$ are projections in $M$ with $E_{N}\left(f_{1}\right)=E_{N}\left(f_{2}\right)=\alpha 1$, where $\alpha \in \Lambda(M, N)$ (respectively $\alpha \in \Lambda(M, N) \cap(0, t)$ ) then there exists a unitary element $u \in N$ such that $u f_{1} u^{*}=f_{2}$.

The proof of this theorem has two parts: existence of values in $\Lambda(M, N)$ and restrictions on the values in $\Lambda(M, N)$. To prove the restriction part we need to introduce a generalization of Jones' tower of projections. This leads us to consider a new class of algebras, generalizing the classical Hecke algebras. We compute some necessary conditions under which these algebras have symmetric nondegenerate representations. These conditions impose restrictions on the existence of generalized towers of projections, in particular on the values in $\Lambda(M, N)$. 
In the case $[M: N] \geq 4$ the information given by the above theorem is incomplete, yet for a special class of subfactors we have a complete characterization of $\Lambda(M, N)$. Namely Jones pointed out in [6] that if for some projection $f \in M, 0<t=\tau(f) \leq 1 / 2$, there is an isomorphism $\theta: f M f \rightarrow(1-f) M(1-f)$ and if one denotes $N=$ $\{x \oplus \theta(x) \mid x \in f M f\}$, then $[M: N]=t^{-1}+(1-t)^{-1} \geq 4$. We call such a subfactor a locally trivial subfactor of $M$. Then we prove, independently of the preceding theorem, that if $N$ is a locally trivial subfactor of $M$ and $\alpha \in \Lambda(M, N)$ with $\alpha \leq 1 / 2$ then $\alpha \leq t$ and the projection $f \in N^{\prime} \cap M$ is the unique one for which $E_{N}(f)=t 1_{M}$. Together with the above theorem this completes the computation of $\Lambda(M, N)$ in this case.

A major interest in understanding the set $\Lambda(M, N)$ comes from the orthogonalization problem for subalgebras of $M$ or, in Jones' terminology, the problem of commuting squares of subalgebras, which is as follows: Let $N \subset M$ be a pair of finite von Neumann algebras. The orthogonalization problem is to find subalgebras $N_{0} \subset M$ for which $E_{N} E_{N_{0}}=E_{N_{0}} E_{N}$. If this relation holds true then we say that $N_{1}=N_{0} \cap N, N_{0}, N, M$ form a commuting square of algebras. This relation between two subalgebras $N_{0}, N \subset M$ has been first considered in [15]. It is important in connection with index problems for subfactors, a fact that has been extensively emphasized in [12]. It turns out that if $N_{1}, N_{0}, N$ are as before then $\Lambda(M, N) \supset \Lambda\left(N_{0}, N_{1}\right)$. Thus, obstructions on $\Lambda(M, N)$ give obstructions on $N_{0}$. As an illustration of this observation we obtain by the preceding theorems a complete solution to the orthogonalization problem for locally trivial subfactors.

1. The set $\Lambda(M, N)$ and the orthogonalization problem. Let $M$ be a finite von Neumann algebra with a normal, finite, faithful trace $\tau, \tau(1)=1$. Denote by $\|x\|_{2}=\tau\left(x^{*} x\right)^{1 / 2}, x \in M$, and by $L^{2}(M, \tau)$ the completion of $M$ in this norm.

Let $N \subset M$ be a von Neumann subalgebra of $M$ always assumed to have the same identity as $M$. Then the closure of $N$ in $L^{2}(M, \tau)$ can be identified with $L^{2}(N, \tau \mid N)$. Let $e_{N}$ be the orthogonal projection of $L^{2}(M, \tau)$ onto $L^{2}\left(N, \tau_{\mid N}\right) \subset L^{2}(M, \tau)$. Let $E_{N}$ be the restriction of $e_{N}$ to $M$ (when regarded as a vector subspace of $L^{2}(M, \tau)$ ). Then $E_{N}$ takes values in $N \subset L^{2}(N, \tau) \subset L^{2}(M, \tau)$ and in fact $E_{N}$ is the unique $\tau$-preserving conditional expectation of $M$ onto $N$.

1.1. Definition. We denote $\Lambda(M, N)=\{\alpha \in \mathbf{R} \mid$ there exists a projection $f_{0} \in M$ such that $\left.E_{N}\left(f_{0}\right)=\alpha 1_{N}\right\}$, the set of all possible 
dimensions of projections which expected on $N$ are scalar multiples of the identity.

1.2. ExAmple. If $N=\mathrm{C} 1$ then $\Lambda(M, N)$ is just the range of the trace $\tau$ on the set of projections $\mathscr{P}(M)$ of $M$. In particular if $M$ is a type $\mathrm{II}_{1}$ factor and $N=\mathrm{C}$ then $\Lambda(M, N)=[0,1]$.

Since a finite factor has a unique trace and any of its automorphisms preserve the trace, we have:

1.3. Proposition. If $M$ is a finite factor and $N \subset M$ is a von Neumann subalgebra then $\Lambda(M, N)$ is an invariant for $N$ up to conjugacy by automorphisms of $M$.

The first invariant to consider for a subfactor $N \subset M$ is the relative commutant $N^{\prime} \cap M$. This invariant is in fact related to $\Lambda(M, N)$ :

1.4. Proposition. If $N \subset M$ are factors then

$$
\Lambda(M, N) \supseteq \tau\left(\mathscr{P}\left(N^{\prime} \cap M\right)\right) .
$$

Proof. If $e \in \mathscr{P}\left(N^{\prime} \cap M\right)$ then for any $y \in N$ we have

$$
y E_{N}(e)=E_{N}(y e)=E_{N}(e y)=E_{N}(e) y
$$

so that $E_{N}(e) \in N^{\prime} \cap N=\mathrm{C}$. Thus $E_{N}(e)=\alpha 1=\tau(e) 1$.

Jones' index [M:N] of the subfactor $N \subset M$ is also related to $\Lambda(M, N)$. Indeed, with the convention $\infty^{-1}=0$, we have by [12]:

\subsection{Proposition. $[M: N]^{-1}=\inf (\Lambda(M, N) \backslash\{0\})$.}

The computation of $\Lambda(M, N)$ is closely related to an important problem about subalgebras of $M$. Namely, we consider subalgebras $N, N_{0} \subset M$ for which the conditional expectations $E_{N}, E_{N_{0}}$ commute.

1.6. Definition. Let $N_{1}, N_{0}, N \subset M$ be von Neumann subalgebras, where $N_{1}=N_{0} \cap N$. If $E_{N_{0}} E_{N}=E_{N} E_{N_{0}}$ we say that $N_{1}, N_{0}, N, M$ form a commuting square of algebras.

This relation between two algebras $N_{0}, N$ was introduced in [15], then in [12] it was shown to be related to index problems for subfactors. More precisely it is shown there that to construct subfactors $N$ of the hyperfinite factor $R$, with trivial relative commutant and given finite index, it is sufficient to find certain commuting squares of finite dimensional algebras. Moreover it is shown in [13] that to find obstructions for the values $[R: N]$ it is sufficient to show that there are 
finite dimensional subalgebras $B_{k} \subset R$ such that $E_{N} E_{B_{k}}=E_{B_{k}} E_{N}$ and $B_{k} \uparrow M$. Other comments on commuting squares can be found in [5].

Commuting squares of algebras and the invariant $\Lambda$ are related by the following:

1.7. Proposition. If $M, N, N_{0}, N_{1}$ is a commuting square of algebras as in 1.6 then $\Lambda\left(N_{0}, N_{1}\right) \subset \Lambda(M, N)$. If in addition all these algebras are factors then $[M: N] \geq\left[N_{0}: N_{1}\right]$.

Proof. Let $e \in \mathscr{P}\left(N_{0}\right), E_{N_{1}}(e)=\alpha 1$. Then $\alpha 1=E_{N_{1}}(e)=E_{N} E_{N_{0}}(e)$ $=E_{N}(e)$. Thus $\alpha \in \Lambda(M: N)$. The second part follows now by 1.5 .

If $N \subset M$ are given, then the problem to find $N_{0} \subset M$ so that $E_{N_{0}} E_{N}=E_{N} E_{N_{0}}\left(=E_{N_{0} \cap N}\right)$ will be called the orthogonalization (or commuting square) problem. The preceding proposition shows on the one hand that if $N \subset M$ are given and we can find a commuting square $N_{1}, N_{0}, N, M$ for which we can compute $\Lambda\left(N_{0}, N_{1}\right)$ then we get information about $\Lambda(M, N)$. On the other hand it shows that if $\Lambda(M, N)$ is known then we get restrictions on the existence of $N_{0}$.

In the case $N \subset M$ are Type $\mathrm{II}_{1}$ factors with finite index $[M: N]$, there are some canonical subalgebras of $M$ which form commuting squares with $N$. Namely Jones proved in [6] that the algebra $M_{1}$ generated by $M$ and $e_{1}=e_{N}$ in $\mathscr{B}\left(L^{2}(M, \tau)\right)$ is a Type $\mathrm{II}_{1}$ factor with the property $M \subset M_{1},\left[M_{1}: M\right]=[M: N]$. Iterating this construction, called the basic construction, one gets recursively an increasing sequence of factors $M_{-1}=N \subset M=M_{0} \subset M_{1} \subset M_{2} \subset \cdots$ with projections $e_{n} \in M_{n}$ so that $\left[M_{n}: M_{n-1}\right]=[M: N], e_{n} x e_{n}=E_{M_{n-2}}(x) e_{n}$, $x \in M_{n-1}, E_{M_{n-1}}\left(e_{n}\right)=\left[M_{n}: M_{n-1}\right]^{-1}, n \geq 1$ ([6]). The sequence $\left\{M_{n}\right\}_{n \geq 1}$ is called the Jones' tower of factors and $\left\{e_{n}\right\}_{n \geq 1}$ the Jones' tower of projections associated with $N \subset M$.

Moreover it is shown in [6] that there exists a subfactor $M_{-2} \subset$ $M_{-1}=N \subset M=M_{0}$ and a projection $e$ in $M$ so that $M$ arises as the basic construction for $M_{-2} \subset M_{-1}$ with $e$ satisfying exe= $E_{M_{-2}}(x) e, x \in M$. Furthermore, the subalgebra $M_{-2} \subset M_{-1}$ and the projection $e \in M$ are unique up to conjugacy by a unitary element in $M_{-1}=N$ by [12]. Iterating this construction, called the downward basic construction, we get a decreasing sequence of factors $\cdots M_{-3} \subset$ $M_{-2} \subset M_{-1}=N \subset M_{0}=M$ and projections $\cdots e_{-3} \in M_{-3}, e_{-2} \in$ $M_{-2}, e_{-1} \in M_{-1}, e_{0}=e \in M=M_{0}$, such that

$$
\begin{aligned}
{\left[M_{-k+1}: M_{-k}\right] } & =[M: N], \quad E_{M_{-k-1}}\left(e_{-k}\right)=\left[M_{-k+1}: M_{-k}\right]^{-1}, \\
e_{-k} x e_{-k} & =E_{M_{-k-2}}(x) e_{-k}, \quad x \in M_{-k-1}, \quad k \geq 0 .
\end{aligned}
$$


The sequence $\left\{M_{-k}\right\}_{k \geq 0}$ is usually called the tunnel of factors and $\left\{e_{-k}\right\}$ the tunnel of projections associated with $N \subset M$.

Let $B_{-k}^{0}=\operatorname{Alg}\left\{1, e_{0}, e_{-1}, \ldots, e_{-k}\right\}, B_{-k}^{-1}=\operatorname{Alg}\left\{1, e_{-1}, e_{-2}, \ldots, e_{-k}\right\}$, $k \geq 1$, and $R_{0}=\overline{\bigcup B_{-k}^{0}}, R_{-1}=\overline{\bigcup B_{-k}^{-1}}$. By [12] $B_{-k}^{0}, B_{-k}^{-1}$ are unique up to conjugacy by a unitary element in $N=M_{-1}$. However the inclusion of $R_{0} \subset M$ is not unique! Yet by Jones' results the inclusion $R_{-1} \subset R_{0}$ only depends on the index [M:N] and it is called Jones pair of factors corresponding to the index $[M: N]$. The next result is implicit in [6] and appears explicitly in [12].

1.8. Proposition. $E_{N} E_{B_{-k}^{0}}=E_{B_{-k}^{0}} E_{N}=E_{B_{-k}^{-1}}$ and $E_{R_{0}} E_{N}=$ $E_{N} E_{R_{0}}=E_{R_{-1}}$.

2. Generalized Hecke algebras and restrictions on $\Lambda(M, N)$. The considerations in this section are motivated by the following observation.

2.1. Proposition. Let $N \subset M$ be a pair of finite factors with finite index and $\lambda=[M: N]^{-1}$. Let $\left\{M_{k}\right\}_{k \geq 1}$ be the associated tower of factors with corresponding tower of projections $\left\{e_{k}\right\}_{k \geq 1}$. Let $\alpha \in \Lambda(M, N)$ and $e_{0} \in M$ be a projection with $E_{N}\left(e_{0}\right)=\alpha 1$. Then $e_{0}, e_{1}, e_{2}, \ldots$ satisfy the axioms:

(i) $e_{k+1} e_{k} e_{k+1}= \begin{cases}\alpha & \text { if } k=0, \\ \lambda & \text { if } k>0\end{cases}$

(ii) $e_{i} e_{j}=e_{j} e_{i}$, if $|i-j| \geq 2$.

Proof. Trivial by the definition.

2.2. Definition. A sequence of self-adjoint projections $\left\{e_{k}\right\}_{k \geq 0}$ acting on a Hilbert space $H$ is called a tower of projections if there are some scalars $\left\{\lambda_{k}\right\}_{k \geq 0}$ such that

(i) The spectrum of $e_{k+1} e_{k} e_{k+1}$ on $e_{k+1} H$ is contained in $\left\{\lambda_{k}, 1\right\}$.

(ii) $e_{i} e_{j}=e_{j} e_{i}$ if $|i-j| \geq 2$.

(iii) A trace $\tau$ on the algebra generated by $\left\{e_{k}\right\}_{k \geq 0}$ is called a Markov trace of the tower if $\tau\left(w_{1} w_{2}\right)=\tau\left(w_{1}\right) \tau\left(w_{2}\right)$ for any words $w_{1}$ in $e_{0}, e_{1}, \ldots, e_{n}$ and $w_{2}$ in $e_{m}, e_{m+1}, \ldots, e_{p}$, where $m>n$.

2.3. LEMMA. The relation (i) in 2.2 is equivalent to each of the following.

(i') $e_{k+1} e_{k} e_{k+1}=e_{k} \wedge e_{k+1}+\lambda_{k}\left(e_{k+1}-e_{k} \wedge e_{k+1}\right)$. 
(i') $\left(\lambda_{k} e_{k+1}-e_{k+1} e_{k} e_{k+1}\right)^{2}=\left(\lambda_{k}-1\right)\left(\lambda_{k} e_{k+1}-e_{k+1} e_{k} e_{k+1}\right)$, in other words $\lambda_{k} e_{k+1}-e_{k+1} e_{k} e_{k+1}$ is a scalar multiple of a projection.

Proof. Trivial by the definition.

As one can easily see the relations (i), (ii) in 2.2 generalize the ones satisfied by the projections obtained from the generators in the semisimple representations of Hecke algebras [7]. This suggests that one should consider suitable more general algebras and study conditions under which they have symmetric representations or equivalently conditions under which there exist corresponding towers of projections on Hilbert space. This leads us to introduce

2.4. Definition. Let $q_{1}, \ldots, q_{n} \in \mathbf{C}$. We denote by $H_{n+1}\left(q_{1}, q_{2}, \ldots, q_{n}\right)$ the complex algebra with identity and generators $g_{1}, g_{2}, \ldots, g_{n}$ satisfying the relations

(1) $g_{k}^{2}=\left(q_{k}-1\right) g_{k}+q_{k}, n \geq k \geq 1$.

(2) $\left(g_{k+1} g_{k} g_{k+1}+g_{k+1} g_{k}+g_{k} g_{k+1}+g_{k+1}+g_{k}+1\right)^{2}$ : $=\left(1+q_{k+1}\right)\left(1+q_{k}+q_{k} q_{k+1}\right)\left(g_{k+1} g_{k} g_{k+1}+g_{k+1} g_{k}+g_{k} g_{k+1}+\right.$ $\left.g_{k+1}+g_{k}+1\right)$

(3) $g_{i} g_{j}=g_{j} g_{i}$ if $|i-j| \geq 2$.

We call this algebra the generalized Hecke algebra with generators $\left(g_{1}, \ldots, g_{n}\right)$ and scalars $\left(q_{1}, \ldots, q_{n}\right)$ (the order is of course important as this algebra is not symmetric in $\left.g_{1}, \ldots, g_{n} !\right)$.

2.5. Proposition. Suppose $q_{k} \neq-1, n \geq k \geq 1$ and let $\lambda_{k}=$ $q_{k+1} /\left(1+q_{k}\right)\left(1+g_{k+1}\right)$ and $e_{k}^{\prime}=1+g_{k} / 1+q_{k}$. Then we have

(a) $e_{k}^{\prime}=e_{k}^{\prime 2}$ and the relation 2.4 , (2) is equivalent to

$$
\left(e_{k+1}^{\prime} e_{k}^{\prime} e_{k+1}^{\prime}-\lambda_{k} e_{k+1}^{\prime}\right)^{2}=\left(1-\lambda_{k}\right)\left(e_{k+1}^{\prime} e_{k}^{\prime} e_{k+1}^{\prime}-\lambda_{k} e_{k+1}^{\prime}\right)
$$

(b) Consider the relation

$$
g_{k+1} g_{k} g_{k+1}=g_{k} g_{k+1} g_{k}, \quad n-1 \geq k \geq 1 .
$$

Then (2") implies the relation (2) and if $H_{n+1}^{\prime}\left(q_{1}, q_{2}, \ldots, q_{n}\right)$ is the algebra defined by the generators $g_{1}, \ldots, g_{n}$ satisfying (1), (2"), (3) then it follows that $q_{1}=q_{2}=\cdots=q_{n}$. Moreover if $q$ denotes this common scalar then $H_{n+1}^{\prime}(q, \ldots, q)$ coincides with the classical Hecke algebra with $n$ generators $H_{n+1}(q)$.

Proof. The first part follows by direct computation. The second part by the identity $g_{k}^{2} g_{k+1} g_{k}=g_{k} g_{k+1} g_{k} g_{k+1}=g_{k+1} g_{k} g_{k+1}^{2}$, which 
follows by $\left(2^{\prime \prime}\right)$. Indeed, this equality yields together with (1) and $\left(2^{\prime \prime}\right): q_{k} g_{k+1} g_{k}=q_{k+1} g_{k+1} g_{k}$, thus $q_{k}=q_{k+1}$.

This proposition shows that whenever $H_{n+1}\left(q_{1}, \ldots, q_{n}\right)$ is represented on a Hilbert space, the elements $1+g_{k} / 1+q_{k}$ become projections. We call a representation on a Hilbert space symmetric if $1+g_{k} / 1+q_{k}$ go into selfadjoint projections. 2.5 above shows that if $e_{k}$ is the image of $1+g_{k} / 1+q_{k}$ under a symmetric representation then $e_{k}$ is a tower of projections as in 2.2 with scalars $\lambda_{k}=$ $q_{k+1} /\left(1+q_{k}\right)\left(1+g_{k+1}\right)$. Thus to find conditions for which such representations exist is equivalent to finding conditions for which generalized towers of projections exist. To do this we need a notation:

2.6. Definition. To any numbers $\left\{\lambda_{k}\right\}_{k \geq 0}$ we associate recursively the numbers $P_{-1} \equiv 1, P_{0} \equiv 1, P_{1}\left(\lambda_{0}\right)=1-\lambda_{0}$,

$$
\begin{aligned}
P_{k+1}\left(\lambda_{0}, \lambda_{1}, \ldots, \lambda_{k}\right)= & P_{k}\left(\lambda_{0}, \ldots, \lambda_{k-1}\right) \\
& -\lambda_{k} P_{k-1}\left(\lambda_{0}, \ldots, \lambda_{k-2}\right), \quad k \geq 1 .
\end{aligned}
$$

Note that if $\lambda_{0}=\lambda_{1}=\cdots=\lambda_{n-1}=\lambda$ then $P_{n}\left(\lambda_{0}, \ldots, \lambda_{n-1}\right)$ coincides with the Jones' polynomial $P_{n}(\lambda)$.

The next result is a generalization of Wenzl's formula in [18]:

2.7. THEOREM. Let $\left\{e_{k}\right\}_{k \geq 0}$ be a tower of projections with corresponding scalars $\left\{\lambda_{k}\right\}_{k \geq 0}$ and let $s_{k}=1-e_{0} \vee \cdots \vee e_{k}, k \geq 0$. If $P_{k}=P_{k}\left(\lambda_{0}, \ldots, \lambda_{k-1}\right) \neq 0$ for all $k \leq n$ then

$$
\begin{array}{ll}
1^{\circ} . & s_{k}=s_{k-1}-\frac{P_{k-1}}{P_{k}} s_{k-1} e_{k} s_{k-1}, \quad \text { for all } 0 \leq k \leq n . \\
2^{\circ} . & \left(s_{n} e_{n+1} s_{n}\right)^{2}=\frac{P_{n+1}}{P_{n}} s_{n} e_{n+1} s_{n} .
\end{array}
$$

Proof. We prove this formula by induction over $k$. Suppose it is true up to some $k<n$. Since $e_{k+1}$ commutes with $e_{0}, e_{1}, \ldots, e_{k-1}$, by induction and 2.3 we get:

$$
\begin{aligned}
e_{k+1} s_{k} e_{k+1}= & e_{k+1} s_{k-1}-\frac{P_{k-1}}{P_{k}} s_{k-1} e_{k+1} e_{k} e_{k+1} s_{k-1} \\
= & e_{k+1} s_{k-1}-\frac{P_{k-1}}{P_{k}} s_{k-1} e_{k} \wedge e_{k+1} s_{k-1} \\
& -\lambda_{k} \frac{P_{k-1}}{P_{k}} s_{k-1}\left(e_{k+1}-e_{k} \wedge e_{k+1}\right) s_{k-1} .
\end{aligned}
$$

Now since $s_{k} s_{k-1}=s_{k}$ and $s_{k} e_{k}=0, s_{k}\left(e_{k} \wedge e_{k+1}\right)=0$ and we get

$$
\begin{aligned}
\left(s_{k} e_{k+1} s_{k}\right)^{2}= & s_{k}\left(e_{k+1} s_{k-1}-\left(P_{k-1} / P_{k}\right) s_{k-1} e_{k} \wedge e_{k+1} s_{k-1}\right. \\
& \left.\quad-\lambda_{k} P_{k-1} / P_{k} s_{k-1}\left(e_{k+1}-e_{k} \wedge e_{k+1}\right) s_{k-1}\right) s_{k} \\
= & \left(1-\lambda_{k} P_{k-1} / P_{k}\right) s_{k} e_{k+1} s_{k}=\left(P_{k+1} / P_{k}\right) s_{k} e_{k+1} s_{k} .
\end{aligned}
$$


Thus $\left(P_{k} / P_{k+1}\right) s_{k} e_{k+1} s_{k}$ is a projection, which implies that

$$
s_{k+1}^{\prime}=s_{k}-\left(P_{k} / P_{k+1}\right) s_{k} e_{k+1} s_{k}
$$

is a projection under $s_{k}$ as well. Moreover $e_{k+1} s_{k+1}^{\prime} e_{k+1}=0$ so that $s_{k+1}^{\prime} e_{k+1}=0$ so that $s_{k+1} \leq s_{k+1}^{\prime}$. But clearly $s_{k+1} s_{k+1}^{\prime}=s_{k+1}$ by definitions, so that $s_{k+1} \leq s_{k+1}^{\prime}$ as well. Thus $s_{k+1}=s_{k+1}^{\prime}=s_{k}$ $P_{k} / P_{k+1} s_{k} e_{k+1} s_{k}$.

This result immediately implies certain restrictions on the possible values of the scalars $\left\{\lambda_{k}\right\}_{k \geq 0}$ for which such a tower exists.

2.8. Corollary. Let $\left\{\lambda_{k}\right\}_{k \geq 0}$ correspond to a tower of projections $\left\{e_{k}\right\}_{k \geq 0}$.

(i) If $P_{k}=P_{k}\left(\lambda_{0}, \ldots, \lambda_{k-1}\right)>0, k \leq n$, and $s_{n} e_{n+1} \neq 0$ then $P_{n+1}=P_{n+1}\left(\lambda_{0}, \ldots ; \lambda_{n}\right) \geq 0$. If $P_{n+1}=0$ then $s_{n} e_{n+1}=0$, or equivalently, $s_{n+1}=s_{n}$.

(ii) If there is a Markov trace $\tau$ on the tower of projections $\left\{e_{k}\right\}_{k \geq 0}$ and if $P_{k} \neq 0, k \leq n$, then

$$
\tau\left(s_{n}\right)=\tau\left(s_{n-1}\right) P_{n+1}\left(\lambda_{0}, \ldots, \lambda_{n-1}, \tau\left(e_{n}\right)\right) / P_{n} .
$$

In particular $\tau\left(s_{n-1}\right) \neq 0$ and $P_{n}>0$ imply

$$
P_{n+1}\left(\lambda_{0}, \lambda_{1}, \ldots, \lambda_{n-1}, \tau\left(e_{n}\right)\right) \geq 0 .
$$

Moreover if $\tau\left(e_{k}\right)=\lambda_{k}, k \leq n$, then $\tau\left(s_{n}\right)=P_{n+1}$.

Proof. If $s_{n} e_{n+1} \neq 0$ then by $2.7,2^{\circ}$ we get $P_{n+1} / P_{n}>0$ so that $P_{n}>0$ implies $P_{n+1}>0$. If $P_{n+1}=0$ but $P_{k}>0, k \leq n$, then $\left(s_{n} e_{n+1} s_{n}\right)^{2}=0$ so that $s_{n} e_{n+1}=0$. If $\tau$ is a Markov trace on $\left\{e_{k}\right\}_{k \geq 0}$ and $P_{k} \neq 0, k \leq n$, then

$$
\begin{aligned}
\tau\left(s_{n}\right) & =\tau\left(s_{n-1}\right)-\frac{P_{n-1}}{P_{n}} \tau\left(s_{n-1} e_{n} s_{n-1}\right) \\
& =\tau\left(s_{n-1}\right)-\frac{P_{n-1}}{P_{n}} \tau\left(s_{n-1} e_{n}\right) \\
& =\tau\left(s_{n-1}\right)-\frac{P_{n-1}}{P_{n}} \tau\left(s_{n-1}\right) \tau\left(e_{n}\right) \\
& =\frac{\tau\left(s_{n-1}\right)}{P_{n}}\left(P_{n}-\tau\left(e_{n}\right) P_{n-1}\right) \\
& =\tau\left(s_{n-1}\right) P_{n+1}\left(\lambda_{0}, \ldots, \lambda_{n-1}, \tau\left(e_{n}\right)\right) / P_{n} .
\end{aligned}
$$

Let us now prove some properties of the Jones' polynomials $P_{n}(\lambda)$. 
2.9. LEMMA.

1。. $P_{n}(\lambda)^{2}-P_{n-1}(\lambda) P_{n+1}(\lambda)=\lambda\left(P_{n-1}(\lambda)^{2}-P_{n-2}(\lambda) P_{n}(\lambda)\right) ; n \geq 1$.

2。. $P_{n}(\lambda)^{2}-P_{n-1}(\lambda) P_{n+1}(\lambda)=\lambda^{n+1}, n \geq 0$.

$3^{\circ}$. If $0 \leq \lambda \leq 1 / 4$ and $0 \leq t \leq 1 / 2$ is so that $t(1-t)=\lambda$ then $P_{n}(\lambda)>0$ for every $n \geq 1$ and the sequence

$$
\left\{\lambda \frac{P_{k-1}(\lambda)}{P_{k}(\lambda)}\right\}_{k \geq 0}
$$

is increasing with initial term $\lambda=\lambda P_{-1} / P_{0}$ and limit point $t$.

$4^{\circ}$.

$$
\begin{aligned}
& P_{n}\left(\left(4 \cos ^{2} \pi /(n+2)\right)^{-1}\right)=0, \\
& P_{n}(\varepsilon)>0 \text { for } \varepsilon<\left(4 \cos ^{2} \pi /(n+2)\right)^{-1} \text { and } \\
& P_{n}(\varepsilon)<0 \text { for }\left(4 \cos ^{2} \pi /(n+2)\right)^{-1}<\varepsilon<\left(4 \cos ^{2} \pi /(n+1)\right)^{-1} .
\end{aligned}
$$

Moreover if $\lambda=\left(4 \cos ^{2} \pi /(n+2)\right)^{-1}$ then the finite sequence

$$
\left\{\lambda \frac{P_{k-1}(\lambda)}{P_{k}(\lambda)}\right\}_{n>k \geq 0}
$$

increases from $\lambda$ to 1 and the finite sequence

$$
\left\{\frac{P_{k}(\lambda)}{P_{k-1}(\lambda)}\right\}_{n \geq k \geq 0}
$$

decreases from 1 to 0.

Proof. We have

$$
\begin{aligned}
& P_{n}(\lambda)^{2}-P_{n-1}(\lambda) P_{n+1}(\lambda) \\
& \quad=P_{n}(\lambda)\left(P_{n-1}(\lambda)-\lambda P_{n-2}(\lambda)\right)-P_{n-1}(\lambda)\left(P_{n}(\lambda)-\lambda P_{n-1}(\lambda)\right) \\
& \quad=\lambda\left(P_{n-1}(\lambda)^{2}-P_{n-2}(\lambda) P_{n}(\lambda)\right) .
\end{aligned}
$$

This proves $1^{\circ}$, then $2^{\circ}$ follows applying $1^{\circ}$ recursively $n$ times until we get

$$
P_{n}(\lambda)^{2}-P_{n-1}(\lambda) P_{n+1}(\lambda)=\lambda^{n}\left(P_{0}^{2}-P_{-1} P_{1}(\lambda)\right)=\lambda^{n+1} .
$$

The first parts of $3^{\circ}$ and $4^{\circ}$ are proved in [6], 4.2.5. Since

$$
\frac{P_{n}(\lambda)}{P_{n+1}(\lambda)}-\frac{P_{n-1}(\lambda)}{P_{n}(\lambda)}=\frac{\lambda^{n+1}}{P_{n+1}(\lambda) P_{n}(\lambda)}
$$

the rest of the statement follows by $2^{\circ}$. 
2.10. LeMMA. If $\lambda_{0}=\alpha, \lambda_{1}=\lambda_{2}=\cdots=\lambda_{n}=\lambda$, then

$$
P_{k+1}\left(\lambda_{0}, \ldots, \lambda_{k}\right)=P_{k}(\lambda)-\alpha P_{k-1}(\lambda),
$$

where $P_{k}(\lambda)$ denotes the Jones' polynomials, $0 \leq k \leq n$.

Proof. Since $P_{k+1}^{\prime}(\alpha, \lambda)=P_{k}(\lambda)-\alpha P_{k-1}(\lambda)$ is a linear combination of Jones' polynomials it follows that $P_{k}^{\prime}, 1 \leq k \leq n$, satisfy the recursive relation $P_{k+1}^{\prime}=P_{k}^{\prime}-\lambda P_{k-1}^{\prime}$. Moreover, since $P_{1}^{\prime}=1-\alpha=P_{1}\left(\lambda_{0}\right), P_{2}^{\prime}=$ $1-\alpha-\lambda=P_{2}\left(\lambda_{0}, \lambda_{1}\right)$ the statement follows by induction.

2.11. THEOREM. Let $\left\{e_{k}\right\}_{k \geq 0}$ be a tower of projections with scalars $\left\{\lambda_{k}\right\}_{k \geq 0}$ and with a Markov trace $\tau$. Suppose $\lambda_{0}=\tau\left(e_{0}\right)=\alpha, \lambda_{k}=$ $\tau\left(e_{k}\right)=\lambda$ for $k \geq 1$. Then $\lambda \in[0,1 / 4] \cup\left\{\left(4 \cos ^{2} \pi /(n+2)\right)^{-1} \mid n \geq 1\right\}$ and $\alpha$ satisfies the condition:

(a) If $\lambda=\left(4 \cos ^{2} \pi /(n+2)\right)^{-1}$ for some $n \geq 1$ then $\alpha=P_{k}(\lambda) / P_{k-1}(\lambda)$ for some $0 \leq k \leq n$.

(b) If $\lambda \leq 1 / 4$ and if $0 \leq t \leq 1 / 2$ is so that $t(1-t)=\lambda$ then $\alpha>1-t$ implies $\alpha=P_{k}(\lambda) / P_{k-1}(\lambda)$ for some $k \geq 0$.

Proof. If $\lambda>1 / 4$ then there exists $n \geq 1$ so that

$$
\left(4 \cos ^{2} \pi /(n+3)\right)^{-1}<\lambda \leq(4 \cos \pi /(n+2))^{-1} \text {. }
$$

If we assume $\lambda \neq\left(4 \cos ^{2} \pi /(n+2)\right)^{-1}$ then it follows that $P_{n}(\lambda)>0$. Considering the tower of projections $\left\{e_{n}\right\}_{n \geq 1}$ with scalars $\lambda=\lambda_{1}=$ $\lambda_{2}=\cdots$, it follows by 2.8 that $P_{n+1}(\lambda) \geq 0$. On the other hand, since $\lambda>\left(4 \cos ^{2} \pi /(n+3)\right)^{-1}$ it follows that $P_{n+1}(\lambda)<0$, a contradiction.

Both in case (a) and in case (b) (when $\alpha>1-t$ ) it follows that for some $k \geq 0$ we have $P_{k+1}(\lambda) / P_{k}(\lambda)<\alpha \leq P_{k}(\lambda) / P_{k-1}(\lambda)$. If $\alpha \neq P_{k}(\lambda) / P_{k-1}(\lambda)$ then $P_{k+1}(\alpha, \lambda, \ldots, \lambda)=P_{k}(\lambda)-\alpha P_{k-1}(\lambda)>0$ so that by 2.8 we get $P_{k+2}(\alpha, \lambda, \ldots, \lambda) \geq 0$, thus $P_{k+1}(\lambda)-\alpha P_{k}(\lambda) \geq 0$, which means that $\alpha \leq P_{k+1}(\lambda) / P_{k}(\lambda)$, a contradiction.

We end this section by reformulating some of the previous results in terms of representations of generalized Hecke algebras. More on these algebras, including computation of indices for associated pairs of subfactors, when a Markov trace is around and in the most simple situations (e.g. under the condition 2.1 (i), (ii)), will be discussed in a forthcoming paper.

2.13. Proposition. $1^{\circ}$. If $H_{n+1}\left(q_{1}, \ldots, q_{n}\right)$ admits a symmetric representation then $\lambda_{k}=q_{k+1} /\left(1+q_{k}\right)\left(1+q_{k+1}\right)$ satisfy the conditions $0 \leq \lambda_{k} \leq 1$. Moreover if the representation admits a Markov trace $\tau$ 
then $\tau\left(e_{k}\right) \leq P_{k}\left(\lambda_{1}, \ldots, \lambda_{k}\right) / P_{k-1}\left(\lambda_{1}, \ldots, \lambda_{k-1}\right)$ for all $k \geq 1$ for which $P_{i}>0$, when $0 \leq i \leq k$.

$2^{\circ}$. If $H_{\infty}\left(q_{0}, q_{1}, \ldots\right)$ admits a symmetric representation $\pi$ with a Markov trace $\tau$ and if $q_{k}=q$ for all $k \geq 1$ then the scalars $\alpha=$ $q /\left(1+q_{0}\right)(1+q), \lambda=q /(1+q)^{2}$ satisfy the conditions

(a) $\lambda \in[0,1 / 4] \cup\left\{4 \cos ^{2} \pi /(n+2) \mid n \geq 1\right\}$

(b) if $\lambda=4 \cos ^{2} \pi /(n+2)$ then $\alpha=P_{k}(\lambda) / P_{k-1}(\lambda)$ for some $0 \leq k \leq$ $n$.

(c) if $\lambda \leq 1 / 4$ and if $t \leq 1 / 2$ is such that $t(1-t)=\lambda$ then $\alpha>1-t$ implies $\alpha=P_{k}(\lambda) / P_{k-1}(\lambda)$ for some $k \geq 0$.

Proof. Follows by 2.4, 2.5, 2.11.

3. Restrictions on $\Lambda(M, N)$ for locally trivial subfactors. For a special class of subfactors we obtain further restrictions on $\Lambda(M, N)$.

3.1. Definition. Let $M$ be a type $\mathrm{II}_{1}$ factor, $f \in M$ a projection of $M$ with $0<\tau(f) \leq 1 / 2$. Suppose there exists an isomorphism $\theta: f M f \rightarrow(1-f) M(1-f)$. Denote $N=\{x \oplus \theta(x) \mid x \in f M f\} . N$ is then called a locally trivial subfactor of $M$.

3.2. Theorem. Suppose $N \subset M$ is a locally trivial subfactor of $M$ like in 3.1. If $\alpha \in \Lambda(M, N), \alpha \leq 1 / 2$, then $\alpha \leq \tau(f)$. Moreover if $f_{0}$ is a projection with $E_{N}\left(f_{0}\right)=\tau(f) 1$ then $f_{0}=f$.

Proof. Let us first observe that if $y \in f M f$ then $E_{N}(y)=t(y \oplus \theta(y))$ and if $y \in(1-f) M(1-f)$ then $E_{N}(y)=(1-t)\left(\theta^{-1}(y) \oplus y\right)$, where $t=\tau(f)$. Suppose $f_{0} \in M$ is so that $E_{N}\left(f_{0}\right)=\alpha 1$ with $\alpha=\tau\left(f_{0}\right)>$ $t=\tau(f)$. Then $f_{1}=f_{0} \wedge(1-f) \neq 0$ and $f_{1} \leq 1-f$ so that $\tau\left(f_{0}\right) 1=$ $E_{N}\left(f_{0}\right) \geq E_{N}\left(f_{1}\right)=(1-t)\left(\theta^{-1}\left(f_{1}\right) \oplus f_{1}\right)$ which implies $\tau\left(f_{0}\right) \geq 1-t \geq$ $1 / 2 \geq \tau\left(f_{0}\right)$. This shows that we must have $\tau\left(f_{0}\right)=\tau(f)=t$.

To prove that we necessarily have $f_{0}=f$ we need first some considerations.

Let $f^{\prime}=f-f_{0} \wedge f, f^{\prime \prime}=$ support $\left((1-f) f_{0}(1-f)\right)$. Then

$$
\begin{aligned}
\tau\left(f^{\prime \prime}\right) & =\tau\left(s\left((1-f) f_{0}(1-f)\right)\right)=\tau\left(s\left((1-f)\left(f_{0}-f_{0} \wedge f\right)(1-f)\right)\right) \\
& =\tau\left(s\left(\left(f_{0}-f_{0} \wedge f\right)(1-f)\left(f_{0}-f_{0} \wedge f\right)\right)\right) \\
& =\tau\left(f_{0}\right)-\tau\left(f_{0} \wedge f\right)=\tau(f)-\tau\left(f_{0} \wedge f\right)=\tau\left(f^{\prime}\right) .
\end{aligned}
$$


We now show that $\theta\left(f_{0} \wedge f\right)=1-f-f^{\prime \prime}$. Indeed by the formula of $E_{N}(y)$ we get:

$$
\begin{aligned}
t 1 & =E_{N}\left(f_{0}\right)=E_{N}\left(f f_{0} f+(1-f) f_{0}(1-f)\right) \\
& \geq E_{N}\left(f_{0} \wedge f+(1-f) f_{0}(1-f)\right) \\
& \geq t \theta\left(f_{0} \wedge f\right)+(1-t)(1-f) f_{0}(1-f) .
\end{aligned}
$$

Thus $\theta\left(f_{0} \wedge f\right)$ is orthogonal to $s\left((1-f) f_{0}(1-f)\right)=f^{\prime \prime}$, so that $\theta\left(f_{0} \wedge f\right) \leq 1-f-f^{\prime \prime}$. To show the other inequality observe that:

$$
\begin{aligned}
E_{N}\left(f_{0}\right)= & t\left(f f_{0} f \oplus \theta\left(f f_{0} f\right)\right) \\
& +(1-t)\left(\theta^{-1}(1-f) f_{0}(1-f)\right) \oplus(1-f) f_{0}(1-f)
\end{aligned}
$$

and since $1-f-f^{\prime \prime}$ is orthogonal to $(1-f) f_{0}(1-f)$ we get

$$
t\left(1-f-f^{\prime \prime}\right)=E_{N}\left(f_{0}\right)\left(1-f-f^{\prime \prime}\right)=t \theta\left(f f_{0} f\right)\left(1-f-f^{\prime \prime}\right) .
$$

Thus $\theta\left(f f_{0} f\right)\left(1-f-f^{\prime \prime}\right)=1-f-f^{\prime \prime}$ which implies that $1-f-f^{\prime \prime}$ is under the spectral projection of $\theta\left(f f_{0} f\right)$ corresponding to $[1, \infty)$. But this spectral projection is equal to $\theta\left(f_{0} \wedge f\right)$. Thus $\theta\left(f_{0} \wedge f\right) \geq 1-f-f^{\prime \prime}$.

We now show that $f_{0} \wedge f=f$. This will end the proof because together with $\tau\left(f_{0}\right)=\tau(f)$ it implies $f_{0}=f$.

To do this note that

$$
\begin{aligned}
\frac{1-t}{t}\left(t-\tau\left(f^{\prime}\right)\right) & =\frac{1-t}{t}\left(\tau\left(f-f^{\prime}\right)\right)=\frac{1-t}{t} \tau\left(f_{0} \wedge f\right) \\
& =\tau\left(\theta\left(f_{0} \wedge f\right)\right)=\tau\left(1-f-f^{\prime \prime}\right)=(1-t)-\tau\left(f^{\prime \prime}\right) \\
& =(1-t)-\tau\left(f^{\prime}\right) .
\end{aligned}
$$

Thus $t-\tau\left(f^{\prime}\right)=t-(t / 1-t) \tau\left(f^{\prime}\right)$ so that $\tau\left(f^{\prime}\right)=0$ which means that $\tau\left(f-f_{0} \wedge f\right)=0$, thus $f_{0} \wedge f=f$.

As one can see, the proof of 3.2 is elementary and uses no results of [6] or [12]. It will be used here to give a complete characterization of $\Lambda(M, N)$ and of the orthogonalization problem for locally trivial subfactors $N \subset M$ (see $\S 6$ below). We mention in this section only a straightforward consequence of the above theorem. This result was proved in [12] by using all the technical machinery developed there. It is important in order to understand the representations of Jones' tower of projections given in [12].

3.3. COROllary. Let $N \subset M$ be a locally trivial subfactor like in 3.1 and $\left\{e_{-k}\right\}_{k \geq 0} \subset M$ the tunnel of projections associated to this pair of factors like in $\S 1$. Then the projection $f\left(\in N^{\prime} \cap M\right)$ belongs to the von Neumann algebra $R_{0}$ generated by $\left\{e_{-k}\right\}_{k \geq 0}$. Moreover if 
$R_{-1}=\left\{e_{-k}\right\}_{k \geq 1}^{\prime \prime}$ then $R_{-1} f=f R_{0} f, R_{-1}(1-f)=(1-f) R_{0}(1-f)$ and $R_{-1}=\left\{x \oplus \theta(x) \mid x \in f R_{0} f\right\}$.

Proof. First we show that $R_{-1}^{\prime} \cap R_{0} \neq \mathbf{C}$. (This part of the proof is taken from [12].) We have $E_{R_{0}}(f) \in R_{-1}^{\prime} \cap R_{0}$. Moreover by the uniqueness of $e_{0} \in M$ up to conjugacy by unitary elements in $M$ we have that $e_{0}=(1-t) p+t q+(t(1-t))^{1 / 2}\left(v+v^{*}\right)$, where $p \leq f, q \leq 1-f$ are projections with

$$
\tau(p)=\tau(q)=t(1-t) \quad\left(=[M: N]^{-1}\right),
$$

$q \stackrel{\text { def }}{=} 1-f-\theta(p)$ and $v^{*} v=p, v v^{*}=q$. Indeed we clearly have $E_{N}\left(e_{0}\right)=t(1-t) 1_{N}$ so that by [12] $e_{0}$ (the first projection in the tunnel) is of this form.

Let $\lambda=t(1-t)$. We have $f e_{0} f=(1-t) p$ so that $\lambda(1-t)=$ $\tau\left(e_{0} f\right)=\tau\left(E_{R_{0}}\left(e_{0} f\right)\right)=\tau\left(e_{0} E_{R_{0}}(f)\right)$ and if $E_{R_{0}}(f)$ would be a scalar multiple of the identity then $\tau\left(e_{0} E_{R_{0}}(f)\right)=\lambda t$, a contradiction. Thus $R_{-1}^{\prime} \cap R_{0} \neq \mathbf{C}$.

Now since $\left[R_{0}: R_{-1}\right]=\tau\left(e_{0}\right)^{-1}=\lambda^{-1}=1 / t+1 / 1-t$ it follows that if $f_{0} \in R_{-1}^{\prime} \cap R_{0}$ with $0<\tau\left(f_{0}\right) \leq 1 / 2$ then $\tau\left(f_{0}\right) \geq \tau(f)$. Indeed because otherwise we would have by [6]:

$$
\begin{aligned}
{\left[R_{0}: R_{-1}\right] } & \geq 1 / \tau\left(f_{0}\right)+1 / \tau\left(1-f_{0}\right) \\
& >1 / \tau(f)+1 / \tau(1-f)=\left[R_{0}: R_{-1}\right]
\end{aligned}
$$

a contradiction.

Now since $\tau\left(f_{0}\right) \geq \tau(f)$ and since

$$
E_{N}\left(f_{0}\right)=E_{N} E_{R_{0}}\left(f_{0}\right)=E_{R_{-1}}\left(f_{0}\right)=\tau\left(f_{0}\right) 1
$$

it follows by 3.2 that $f_{0}=f$. The rest of the statement is now trivial.

3.4. Corollary. Let $\left\{\boldsymbol{e}_{-k}\right\}_{k \geq 0}$ be the Jones' projections with scalar $\lambda$ and let $R_{0}=\left\{e_{-k}\right\}_{k \geq 0}^{\prime \prime}, R_{-1}=\left\{e_{-k}\right\}_{k \geq 1}^{\prime \prime}$. If $\lambda<1 / 4$ then $R_{-1}$ is $a$ locally trivial subfactor of $R_{0}$.

4. Values in $\Lambda(M, N)$ and unitary conjugacy. By Jones' results in [6], if $N \subset M$ has finite index and $\lambda=[M: N]^{-1}$ then $\lambda \in \Lambda(M, N)$. We show now how we can generate a whole sequence of values in $\Lambda(M, N)$ starting from $\lambda$. In what follows we use the notation $M^{\beta}$ for the $\beta$-amplification of $M$ by $\beta>0$ (for the definition see e.g. [12]). As usual $M_{1} \supset M$ is the factor obtained by the basic construction for $N \subset M$. 
4.1. Proposition. $1^{\circ}$. If $\alpha \in \Lambda(M, N)$ then $1-\alpha \in \Lambda(M, N)$.

$2^{\circ}$. If $\alpha \in \Lambda(M, N), \alpha \neq 0,1$, then $\lambda / \alpha \in \Lambda\left(M_{1}^{\alpha}, M^{\alpha}\right)$ and $\lambda / 1-\alpha \in \Lambda\left(M_{1}^{1-\alpha}, M^{1-\alpha}\right)$.

Proof. If $f_{0} \in M$ is such that $E_{N}\left(f_{0}\right)=\alpha 1$ then $M^{\alpha}=f_{0} M f_{0} \subset$ $f_{0} M_{1} f_{0}=M_{1}^{\alpha}$ and $f_{1}=\alpha^{-1} f_{0} e_{1} f_{0} \in M_{1}^{\alpha}$ is a projection $\left(e_{1} \in M_{1}\right.$ is the projection with $\left.\tau\left(e_{1}\right)=\lambda, e_{1} x e_{1}=E_{N}(x) e_{1}, x \in M\right)$. Indeed we have $\left(f_{0} e_{1} f_{0}\right)^{2}=\alpha f_{0} e_{1} f_{0}$. It follows that the trace of $f_{1}$ in $f_{0} M_{1} f_{0}$ is $\lambda / \alpha$ and $E_{M^{\prime \prime}}\left(f_{1}\right)=\lambda / \alpha 1$. Taking $1-\alpha$ instead of $\alpha$ completes the proof.

4.2. Corollary. If $P_{k}(x), k \geq-1$, denote the Jones' polynomials, as usual, and if $P_{k}\left([M: N]^{-1}\right)>0$ for $1 \leq k<n$ then $P_{k-1}([M$ : $\left.N]^{-1}\right) /[M: N] P_{k}\left([M: N]^{-1}\right) \in \Lambda(M, N)$ for $0 \leq k<n$.

Proof. We prove this by induction. Suppose we proved that $P_{k-1}\left([M: N]^{-1}\right) /[M: N] P_{k}\left([M: N]^{-1}\right) \in \Lambda(M, N)$ for $k \leq m$.

Let $\alpha=P_{m-1}\left([M: N]^{-1}\right) /[M: N] P_{m}\left([M: N]^{-1}\right)$ and $\beta=(1-\alpha)^{-1}$. Let $N^{\beta} \subset M^{\beta}$ be the $\beta$ amplification of $N \subset M$ and let $\tilde{N}_{1} \subset N^{\beta}$ be a subfactor so that $M^{\beta}$ be the extension of $N^{\beta}$ by $\tilde{N}_{1}$. Let also $\tilde{e}_{0} \in M^{\beta}$ be the projection implementing the conditional expectation of $N^{\beta}$ onto $\tilde{N}_{1}$. Since $\left[N^{\beta}: \tilde{N}_{1}\right]=\left[M^{\beta}: N^{\beta}\right]=[M: N]$ it follows by the induction hypothesis that $\alpha \in \Lambda\left(N^{\beta}, \tilde{N}_{1}\right)$. Let $f_{1} \in N^{\beta}$ be so that $E_{\tilde{N}_{1}}\left(f_{1}\right)=\alpha 1$. By 4.1 it follows that $\lambda / 1-\alpha \in \Lambda\left(\left(M^{\beta}\right)^{1-\alpha},\left(N^{\beta}\right)^{1-\alpha}\right)$. But $\left(M^{\beta}\right)^{1-\alpha}=M,\left(N^{\beta}\right)^{1-\alpha}=N$. Since

$$
\lambda / 1-\alpha=P_{m}\left([M: N]^{-1}\right) /[M: N] P_{m+1}\left([M: N]^{-1}\right),
$$

the result follows.

To solve the conjugacy problem for the values $\alpha=\lambda P_{n-1}(\lambda) / P_{n}(\lambda)$ we need to prove another formula for the projections $f_{0} \in M$ for which $E_{N}\left(f_{0}\right)=\alpha 1$.

4.3 Lemma. Let $\lambda=[M: N]^{-1},\left\{M_{n}\right\}_{n \geq 1},\left\{e_{n}\right\}_{n \geq 1}$ be the Jones' tower of factors and respectively projections associated with $N \subset M$ as in $\S 1$. Let $0 \leq k$ be so that $\lambda P_{i-1}(\lambda) / P_{i}(\lambda)>0$ for all $i \leq k$ and let $\alpha=\lambda P_{k-1}(\lambda) / P_{k}(\lambda)$. Let $f_{0} \in M$ be a projection such that $E_{N}\left(f_{0}\right)=\alpha 1$ (cf. 4.2). If we denote $p_{i}=\left(1-e_{1}\right) \wedge \cdots \wedge\left(1-e_{i}\right), p_{0, i}=f_{0} \wedge\left(1-e_{1}\right) \wedge$ $\cdots \wedge\left(1-e_{i}\right)$ then we have:

(i) $f_{0}=\lambda^{-k} P_{k-1}(\lambda) E_{M}\left(p_{0, k}\right)$. 
(ii) $p_{j} \in N^{\prime} \cap M_{k}, j \leq k, p_{0, k} \in p_{k} M_{k} p_{k}$ and we have $E_{N p_{k}}\left(p_{0, k}\right)=$ $\left[p_{k} M_{k} p_{k}: N p_{k}\right]^{-1} 1$.

Proof. Since $1-f_{0}, e_{1}, e_{2}, \ldots$ satisfy the axioms of a generalized tower of projections with scalars $1-\lambda P_{k-1}(\lambda) / P_{k}(\lambda), \lambda, \lambda, \ldots$ it follows by 2.7 that $p_{0, k}=p_{0, k-1}-\left(P_{k+1}(\lambda) / \lambda P_{k}(\lambda)\right) p_{0, k-1} e_{k} p_{0, k-1}$. Thus $E_{M_{k-1}}\left(p_{0, k}\right)=\lambda P_{k+1}(\lambda) / P_{k}(\lambda) p_{0, k-1}$ and (i) follows now by induction. In particular this shows that $E_{N}\left(p_{0, k}\right)$ is a scalar multiple of the identity. Since clearly $p_{k} \in N^{\prime} \cap M_{k}$ and $p_{k} \geq p_{0, k}$ it follows that $E_{N_{p_{k}}}\left(p_{0, k}\right)$ is also a scalar multiple of the identity and in fact that $E_{N_{p_{k}}}\left(p_{0, k}\right)=E_{N}\left(p_{0, k}\right) p_{k} \tau\left(p_{k}\right)^{-1}=\tau\left(p_{0, k}\right) \tau\left(p_{k}\right)^{-1} 1$. By 2.10 we have $\tau\left(p_{0, k}\right)=P_{k}(\lambda)-(1-\alpha) P_{k-1}(\lambda)$. Thus we get $\tau\left(p_{0, k}\right) / \tau\left(p_{k}\right)=$ $\left(P_{k}(\lambda)^{2}-P_{k+1}(\lambda) P_{k-1}(\lambda)\right) / P_{k}(\lambda)^{2}$. On the other hand we have by 4.2 .5 in [6] the formula $\left[p_{k} M_{k} p_{k}: N_{p_{k}}\right]=\left[M_{k}: N\right] \tau\left(p_{k}\right) \tau^{\prime}\left(p_{k}\right)$. Since $p_{k}$ is generated by projections $e_{i}, 1 \leq i \leq k$, and since $\tau^{\prime}$ coincides with $\tau$ on the algebra generated by such projections, it follows that $\left[p_{k} M_{k} p_{k}: N p_{k}\right]=\lambda^{-k-1} P_{k}(\lambda)^{2}$. Now (ii) follows by 2.9 .

4.4. Corollary. Let $f_{1}, f_{2} \in M$ be projections with the property that $E_{N}\left(f_{1}\right)=E_{N}\left(f_{2}\right)=\alpha 1$. Suppose $\alpha=\lambda P_{k-1}(\lambda) / P_{k}(\lambda)$ for some $k \geq 0$ for which $P_{i}(\lambda)>0, i \leq k$. Then there exists a unitary element $w \in N$ such that $w f_{1} w^{*}=f_{2}$.

Proof. Let $p_{i, k}=f_{i} \wedge\left(1-e_{1}\right) \wedge \cdots \wedge\left(1-e_{k}\right) \in M_{k}, \quad i=1,2$, $p_{k}=\left(1-e_{1}\right) \wedge \cdots \wedge\left(1-e_{k}\right) \in N^{\prime} \cap M_{k}$ as in 4.3. By the preceding lemma we have $E_{N p_{k}}\left(p_{i, k}\right)=\left[p_{k} M_{k} p_{k}: M_{p_{k}}\right]^{-1} 1$. By 1.6 in [12] it follows that there exists a unitary element $w_{0} \in N p_{k}$ so that $w_{0} p_{1, k} w_{0}^{*}=p_{2, k}$. But then $w_{0}=w p_{k}$ for a unitary element $w$ in $M$. Thus $w p_{1, k} w^{*}=p_{2, k}$ and applying the conditional expectation $E_{M}$ to both sides, we get by 4.3, (i) $w f_{1} w^{*}=f_{2}$.

We will now point out some other properties of the projections whose dimension relative to $N$ are scalars of the form $\lambda P_{k-1}(\lambda) / P_{k}(\lambda)$, $\lambda=[M: N]^{-1}$.

4.5. Proposition. If $f_{0} \in M$ is so that $E_{N}\left(f_{0}\right)=\alpha 1_{N}$ with $\alpha=$ $\lambda P_{k-1}(\lambda) / P_{k}(\lambda)$ for some $k$ for which $P_{i}(\lambda)>0,1 \leq i \leq k$, then there exists a subfactor $N_{1} \subset N$ of index $\left[N: N_{1}\right]=\lambda^{-k-1} P_{k}(\lambda)^{2}$ so that $f_{0} \in N_{1}^{\prime} \cap M$.

Proof. Using the notations in the proof of 4.3 we have that $N p_{k} \subset$ $p_{k} M_{k} p_{k}$ has index $\lambda^{-k-1} P_{k}(\lambda)$ and $p_{0, k}=f_{0} \wedge p_{k}$ has the property 
that $E_{N p_{k}}\left(p_{0, k}\right)=\left[p_{k} M_{k} p_{k}: N p_{k}\right]^{-1}=\lambda^{k+1} / P_{k}(\lambda)^{2}$. Thus there exists a subfactor $N_{1} \subset N$ so that $p_{k} M_{k} p_{k}$ and $p_{0, k}$ come from the basic construction for the inclusion $N_{1} p_{k} \subset N p_{k}$. It follows in particular that if $y_{1} \in N_{1}$ then $y_{1} p_{k} p_{0, k}=p_{0, k} y_{1} p_{k}$. Since $p_{0, k} \leq p_{k}$ and $\left[p_{k}, y_{1}\right]=0$ it follows that $\left[p_{0, k}, y_{1}\right]=0$. Thus $0=\left[E_{M}\left(p_{0, k}\right), y_{1}\right]=$ $\left[P_{k+1}(\lambda) / P_{k}(\lambda) f_{0}, y_{1}\right]$.

4.6. REMARK $1^{\circ}$. We mention that the numbers $\lambda P_{k-1}(\lambda) / P_{k}(\lambda)$ coincide with the traces of the projections coming from the generators of the Hecke algebras when regarded in their symmetric semisimple representations which have a Markov trace. More precisely let $H_{n+1}(q)$ be the Hecke algebra with $n$ generators $g_{1}, \ldots, g_{n}$ and let $e_{i}=1+g_{i} / 1+q$. Suppose $\pi$ is a representation of $H_{n+1}(q)$ on a Hilbert space so that $\pi\left(e_{i}\right)^{*}=\pi\left(e_{i}\right)$ are self-adjoint projections. Denote by $\lambda=q /(1+q)^{2}$. It is shown in [7] that $\left\{e_{l}\right\}_{i \geq 1}$ then satisfy 2.2 (i), (ii) with $\lambda_{1}=\lambda_{2}=\cdots=\lambda$. Moreover [7], [17] it follows that $\lambda$ can only take the values

$$
\lambda \in(0,1 / 4] \cup\left\{\left(4 \cos ^{2} \pi /(n+2)\right)^{-1} \mid n \geq 1\right\} .
$$

If in addition there is a Markov trace $\tau=\tau_{\lambda, k}$ on $\left\{\pi\left(e_{i}\right)\right\}_{i \geq 1}$ (i.e., $\tau$ satisfies 2.2 (iii)) then we must have one of the following: if $\lambda=$ $(4 \cos 2 \pi /(n+2))^{-1}, n \geq 1$, then $\tau\left(e_{i}\right)=\lambda P_{k-1}(\lambda) / P_{k}(\lambda)$ for some $0 \leq k<n$ and all $i \geq 1$; if $\lambda \leq 1 / 4$ then $\tau\left(e_{i}\right)=\lambda P_{k-1}(\lambda) / P_{k}(\lambda)$ for some $k \geq 0$ and all $i \geq 1$.

$2^{\circ}$. It seems then legitimate to ask: is there a "generalized basic construction" which associates the projection $f_{0} \in M$, with $E_{N}\left(f_{0}\right)=$ $\lambda P_{k-1}(\lambda) / P_{k}(\lambda)$, to the inclusion $M \subset M$ in an as canonical as possible way? Propositions 4.3 and 4.5 give a partially positive answer to this question. More precisely, if we take the factors $M_{-k-2} \subset M_{-k-1} \subset$ $\cdots \subset N=M_{-1} \subset M_{0}=M \subset M_{1} \subset \cdots \subset M_{k}$ and denote $p_{k}=$ $\left(1-e_{1}\right) \wedge \cdots \wedge\left(1-e_{k}\right), p_{k}^{\prime}=\left(1-e_{-k}\right) \wedge \cdots \wedge\left(1-e_{-1}\right)$ then one can only see that $p_{k}^{\prime} p_{k} M_{k} p_{k} p_{k}^{\prime}$ comes from the basic construction for the inclusion $p_{k} p_{k}^{\prime} M_{-k-1} p_{k}^{\prime} p_{k} \subset p_{k} p_{k}^{\prime} N p_{k}^{\prime} p_{k}$. If $\tilde{e}$ is the projection implementing the conditional expectation of $p_{k} p_{k}^{\prime} N p_{k}^{\prime} p_{k}$ onto $p_{k} p_{k}^{\prime} M_{-k-2} p_{k}^{\prime} p_{k}$ then we get

$$
f_{0} p_{k}^{\prime}=\frac{P_{k}(\lambda)}{P_{k+1}(\lambda)} E_{M}(\tilde{e})
$$

Thus, once the subfactors $\left\{M_{-l-2}\right\}_{k \geq i \geq 0}$ of the tunnel are fixed, $f_{0} p_{k}^{\prime}$ is canonical.

$3^{\circ}$. If $N=R_{-1} \subset R_{0}=M$ is the Jones' pair of factors of index $\lambda^{-1}$ then $R_{-1} p_{k} \subset p_{k} R_{k} p_{k}$ has the same index as does the pair 
of hyperfinite factors $P_{-1} \subset P_{0}$ associated to the representation $\pi_{\lambda}$ and trace $\tau_{\lambda, k}$ of the infinite Hecke algebra (cf [17]). Also we have $\left(R_{-1} p_{k}\right)^{\prime} \cap p_{k} R_{k} p_{k}=\mathrm{C}$ by an unpublished result of Skau. The question then arises: does the inclusion $R_{-1} p_{k} \subset p_{k} R_{k} p_{k}$ coincide with $P_{-1} \subset P_{0}$ ? Wenzl computed the towers of higher relative commutants for $R_{-1} p_{k} \subset p_{k} R p_{k}$ and for his pair of factors in [17] and showed that they don't coincide. Thus $R_{-1} p_{k} \subset p_{k} R p_{k}$ is in fact not isomorphic to the pair of factors $P_{-1} \subset P_{0}$ in [17].

4.7. Proposition. Let $\lambda \in\left\{\left(4 \cos ^{2} \pi /(n+2)\right)^{-1} \mid n \geq 1\right\} \cup[4, \infty)$ and let $R_{-1} \subset R_{0}$ be Jones' pair of factors of index $\lambda^{-1}$, as in [6]. Let $R_{0} \stackrel{e_{1}}{\subset} R_{1} \stackrel{e_{2}}{\subset} R_{2} \stackrel{e_{3}}{\complement} \ldots$ be the associated tower of projections and $p_{k}=\left(1-e_{1}\right) \wedge \cdots \wedge\left(1-e_{k}\right) \in R_{-1}^{\prime} \cap R_{k}$ as in 4.3. Then

$$
\left[p_{k} R_{k} p_{k}: R_{-1} p_{k}\right]=\lambda^{-k-1} P_{k}(\lambda)^{2}
$$

If in addition $\lambda=\left(4 \cos ^{2} \pi /(n+2)\right)^{-1}$ then

$$
\lambda^{-k-1} P_{k}(\lambda)^{2}=\left(\sin ^{2}(k+2) \pi /(n+2)\right) / \sin ^{2} \pi /(n+2)
$$

and $\left(R_{-1} p_{k}\right)^{\prime} \cap p_{k} R_{k} p_{k}=$ C. Moreover, if we put $q /(1+q)^{2}=\lambda$ then $\lambda^{-k-1} P_{k}(\lambda)=\left(\left(1-q^{k}\right) / q^{(k-1)} / 2(1-q)\right)^{2}$.

Proof. Trivial by 4.3 , by the formula $P_{k}(\lambda)^{2}-P_{k+1}(\lambda) P_{k-1}(\lambda)=\lambda^{k+1}$ and by

$$
\begin{aligned}
& P_{k}\left(\left(4 \cos ^{2} \pi /(n+2)\right)^{-1}\right) \\
& \quad=(\sin (k+2) \pi /(n+2)) / 2^{k+1} \cos ^{k+1} \pi /(n+2) \sin \pi /(n+2)
\end{aligned}
$$

(cf. [6]). The trivial relative commutant is a consequence of the above mentioned unpublished result of Skau. The rest is simple computation.

4.8. Corollary. If $\lambda=\left(4 \cos ^{2} \pi /(n+2)\right)^{-1}$ then the inclusion $R_{-1} p_{k} \subset p_{k} R_{k} p_{k}$ has the same index and relative commutant as the pair of factors $A \subset B$ of 4.2 in [17] associated with appropriate symmetric representations of Hecke algebras.

\section{Computation of $\Lambda(M, N)$.}

5.1. TheOREM. Suppose $[M: N]=4 \cos ^{2} \pi /(n+2)$ for some $n \geq$ 1. Then $\Lambda(M, N)=\left\{P_{k}\left([M: N]^{-1}\right) / P_{k-1}\left([M: N]^{-1}\right) \mid n \geq k \geq 0\right\}$. 
Moreover if $f_{1,2} \in M$ are projections such that $E_{N}\left(f_{1}\right)=E_{N}\left(f_{2}\right)=\alpha 1_{N}$ then there exists a unitary element $u$ in $N$ such that $u f_{1} u^{*}=f_{2}$.

Proof. By 4.2 we have that $P_{k}\left([M: N]^{-1}\right) / P_{k-1}\left([M: N]^{-1}\right) \in$ $\Lambda(M, N)$ for each $n \geq k \geq 0$ and by 4.4 any two projections expected on the same scalar on $N$ are conjugate by a unitary element in $M$. By 2.8 these values are the only ones that may appear in $\Lambda(M, N)$. The conjugacy follows by 4.4 .

5.2. TheOREM. Suppose $[M: N] \geq 4$ and let $0<t \leq 1 / 2$ be so that $t(1-t)=[M: N]^{-1}$. Then

$$
\begin{aligned}
& \Lambda(M, N) \cap[0, t] \\
& \quad=\{0, t\} \cup\left\{P_{k-1}\left([M: N]^{-1}\right) /[M: N] P_{k}\left([M: N]^{-1}\right) \mid k \geq 0\right\}
\end{aligned}
$$

and

$$
\begin{aligned}
& \Lambda(M, N) \cap[1-t, 1] \\
& \quad=\{1-t\} \cup\left\{P_{k}\left([M: N]^{-1}\right) / P_{k-1}\left([M: N]^{-1}\right) \mid k \geq 0\right\} .
\end{aligned}
$$

Moreover if $\alpha \neq t, 1-t$ is in either of these sets and $f_{1,2}$ are projections in $M$ with $E_{N}\left(f_{1}\right)=E_{N}\left(f_{2}\right)=\alpha 1_{N}$ then there exists a unitary element $u \in N$ so that $u f_{1} u^{*}=f_{2}$.

Proof. The existence of values $\alpha=P_{k}\left([M: N]^{-1}\right) / P_{k-1}\left([M: N]^{-1}\right)$ follows by 4.2 and the unitary conjugacy by 4.4. The other values are of the form $1-\alpha$ or $\alpha=t, 1-t$. That $t$ is in $\Lambda(M, N)$ follows by 1.7, $1.8,3.4$. The obstructions follow by 2.8 .

5.3. TheOREM. Let $N \subset M$ be locally trivial subfactor as in 3.1 . Then

$$
\begin{aligned}
\Lambda(M, N)= & \{0\} \cup\left\{P_{k-1}\left([M: N]^{-1}\right) /[M: N] P_{k}\left([M: N]^{-1}\right) \mid k \geq 0\right\} \\
& \cup\{t, 1-t\} \cup\left\{P_{k}\left([M: N]^{-1}\right) / P_{k-1}\left([M: N]^{-1}\right) \mid k \geq 0\right\} .
\end{aligned}
$$

Moreover, given any $\alpha$ in $\Lambda(M, N)$ and $f_{1,2} \in M$ projections with $E_{N}\left(f_{1}\right)=E_{N}\left(f_{2}\right)=\alpha 1_{N}$ there exists a unitary element $u \in N$ such that $u f_{1} u^{*}=f_{2}$.

Proof. By 5.2 and 3.3 the statement follows.

As one can see, the information about $\Lambda(M, N)$ that we obtained here depends entirely on the index $[M: N]$. In the case $[M: N]>4$ this information is incomplete, in the sense that we don't know what 
happens in the interval $(t, 1-t)$. As in the case of the index problems, it seems that the case $N^{\prime} \cap M=\mathrm{C}$ is of most interest. Yet there are a number of other problems that should be clarified and that we mention here:

5.4. Problems. 5.4.1. If $p \in N$ is a projection, is it true that $\Lambda(p M p, p N p)=\Lambda(M, N)$ ?

5.4.2. Is $\Lambda(M, N)$ equal to $\Lambda\left(M_{1}, M\right)$ ? Is this true if $N^{\prime} \cap M=\mathrm{C}$ ?

5.4.3. Is it true that if $N^{\prime} \cap M=\mathrm{C},[M: N]>4$ then $\Lambda(M, N) \cap$ $(t, 1-t) \neq \varnothing ?$ (Where $t(1-t)=[M: N]^{-1}$.)

5.4.4. If $[M: N]>4$ and $f, f_{0} \in M$ are projections with $E_{N}(f)=$ $E_{N}\left(f_{0}\right)=t 1_{N}$ does there exist $u \in N$ with $u f u^{*}=f_{0}$ ? Does there always exist a tunnel of projections $\left\{e_{-k}\right\}_{k \geq 0} \subset M$ associated with the inclusion $N \subset M$ so that $f \in R_{-1}^{\prime} \cap R_{0}$ ?

5.4.5. If $N^{\prime} \cap M=C$ and $f_{1,2}$ are projections in $M$ with $E_{N}\left(f_{1}\right)=$ $E_{N}\left(f_{2}\right)=\alpha 1$ for some $\alpha \in \Lambda(M, N)$, does it follow that $f_{1}, f_{2}$ are conjugate by a unitary element in $N$ ? (Of course in the cases uncovered by $5.1,5.2$.)

5.4.6. Can $\Lambda(M, N)$ be uncountable? Is it always closed? Can it contain an interval?

In connection with 5.4.1 we should mention here one of Jones' problems in [6]. He asked there whether $M$ splits the hyperfinite $\mathrm{II}_{1}$ factor $R$, i.e., $M \simeq M \otimes R$, then there exists a hyperfinite $\mathrm{II}_{1}$ subfactor $R$ in $N$ so that $N=R \vee\left(R^{\prime} \cap N\right)$ and $M=R \vee\left(R^{\prime} \cap M\right)$. That $N$ splits $R$, if $M$ does, was proved in [12]. In the case $M$ itself is the hyperfinite $\mathrm{II}_{1}$ factor $R$ it follows by Connes' fundamental theorem that $N$ is also isomorphic to $R$. Then for a special but most interesting class of subfactors $N \subset R$, called subfactors with finite depth, Ocneanu announced in [11] results which in particular imply that the inclusion $N \subset R$ splits in this case the hyperfinite $\mathrm{II}_{1}$ factor. In case the inclusion $N \subset M$ splits $R$ the inclusions $p N p \subset p M p$ is isomorphic to $N \subset M$ for any projection $p$ in $N$. There is of course a natural invariant to consider for the inclusion $N \subset M$ along these lines.

5.4.7. Definition [14]. Let $M$ be a type $\mathrm{II}_{1}$ factor, $N \subset M$ a type $\mathrm{II}_{1}$ subfactor (not necessarily with finite index). Let $\mathscr{F}(M, N)=\{\beta>0 \mid$ $N^{\beta} \subset M^{\beta}$ is isomorphic to $\left.N \subset M\right\}$. We call this set the fundamental group of the inclusion $N \subset M$ (it is clearly a multiplicative subgroup of $\mathbf{R}_{+}^{*}$ ).

It is easy to see that $\mathscr{F}(M, N)$ is invariant up to conjugacy of $N$ by automorphisms of $M$ and $\mathscr{F}(M, M)=\mathscr{F}(M)$ is just the fundamental group of $M$. If $N \subset M$ splits $R$ then we have $\mathscr{F}(M, N)=\mathbf{R}_{+}^{*}$. In other 
situations however it seems very difficult to compute (as it is $\mathscr{F}(M)$ !). We mention two more problems related to this definition:

5.4.8. Find a type $\mathrm{II}_{1}$ subfactor $N \subset R$, of finite or infinite index, so that $\mathscr{F}(R, N) \neq \mathbf{R}_{+}^{*}$.

5.4.9. If $M$ splits $R$ and $N \subset M$ is a locally trivial subfactor, does it follow that $N \subset M$ splits $R$ ?

To handle the values $\Lambda(M, N) \cap(t, 1-t)$ (in the case $[M: N]^{-1}=$ $t(1-t)<1 / 4)$ seems very difficult. We can however deduce from 5.1 that in case the inclusion $N \subset M$ is isomorphic to the inclusions $p N p \subset p M p$ obtained by reducing it with certain projections of $M$, then from one given value in $\Lambda(M, N)$ we can get a whole sequence of values in $\Lambda(M, N)$, with $t$ (or $1-t)$ as limit point.

5.5. Proposition. Let $N \subset M$ be type $\mathrm{II}_{1}$ factors with $4<$ $[M: N]<\infty$. Suppose $\alpha \in \Lambda(M, N), t<\alpha \leq 1 / 2$ where $t(1-t)=$ $[M: N]^{-1}=\lambda$. Let $\left\{\alpha_{n}\right\}_{n \geq 0}$ be the sequence of elements defined recursively by $\alpha_{0}=\alpha, \alpha_{n+1}=\lambda / 1-\alpha_{n}, n \geq 0$. If the inclusion $N \subset M$ splits $R$ (i.e., there exists $R \subset N$ with $\left.N=R \vee\left(R^{\prime} \cap N\right), M=R \vee\left(R^{\prime} \cap M\right)\right)$ then $\left\{\alpha_{2 n}\right\}_{n \geq 0} \subset \Lambda(M, N),\left\{\alpha_{2 n+1}\right\}_{n \geq 0} \subset \Lambda\left(M_{1}, M\right)$ and $\alpha_{n}$ is a sequence decreasing to $t$. More generally if $\lambda / 1-\alpha-\lambda$ is in the fundamental group $\mathscr{F}(M, N)$ of the inclusion $N \subset M$ then $\left\{\alpha_{2 n}\right\}_{n \geq 0} \subset \Lambda(M, N)$.

Proof. By 5.1 if $\alpha_{n} \in \Lambda(M, N)$ then $\alpha_{n+1} \in \Lambda\left(M_{1}^{1-\alpha_{n}}, M^{1-\alpha_{n}}\right)$, $\alpha_{n+2} \in \Lambda\left(M_{2}^{\left(1-\alpha_{n}\right)\left(1-\alpha_{n+1}\right)}, M_{1}^{\left(1-\alpha_{n}\right)\left(1-\alpha_{n+1}\right)}\right)$. But by 1.5 in [12] we have $M_{2}^{\lambda} \supset M_{1}^{\lambda}$ isomorphic to $M \supset N$. The fact that $\alpha_{n}$ is decreasing to $t$ follows by induction and by the relation $\lim \alpha_{n+1}=$ $\lambda / 1-\lim \alpha_{n}$.

\section{Application to the orthogonalization problem.}

6.1. TheOREM. Let $N$ be a subfactor of finite index of the type $\mathrm{II}_{1}$ factor $M$. Suppose $N_{1} \subset N_{0}$ are subfactors of $M$ which form a commuting square with $N, M$, i.e., $E_{N} E_{N_{0}}=E_{N_{0}} E_{N}=E_{N_{1}}$. If $[M: N]=4 \cos ^{2} \pi /(n+2)<4$, for some $n \geq 1$, then $\left[N_{0}: N_{1}\right]=$ $[M: N] P_{k}\left([M: N]^{-1}\right) / P_{k-1}\left([M: N]^{-1}\right)$ for some $0 \leq k<n$. If $[M: N] \geq 4$ and $t \leq 1 / 2$ is such that $[M: N]^{-1}=t(1-t)$ then either $\left[N_{0}: N_{1}\right]=[M: N] P_{k}\left([M: N]^{-1}\right) / P_{k-1}\left([M: N]^{-1}\right)$ for some $k \geq 0$ or $\left[N_{0}: N_{1}\right] \leq t^{-1}$.

Proof. By 1.7 we have $\Lambda\left(N_{0} N_{1}\right) \subset \Lambda(M, N)$. Since $\left[N_{0}: N_{1}\right]^{-1} \in$ $\Lambda\left(N_{0}, N_{1}\right)$ the statement follows by 5.1 and 5.2 . 
There are of course other restrictions coming from the condition $\Lambda\left(N_{0}, N_{1}\right) \subset \Lambda(M, N)$ and from 5.1, 5.2, conditions that in fact imply that in the case $[M: N]$ is "small" one almost always have $\left[N_{0}: N_{1}\right]=[M: N]$. We leave the detailed analysis of those situations as an exercise to the reader. There is one case when the description of commuting squares is complete:

6.2. THEOREM. Let $M$ be a type $\mathrm{II}_{1}$ factor $f \in M$ a projection of trace $0<t<1 / 2$. Suppose there exists an isomorphism $\theta: f M F \rightarrow$ $(1-f) M(1-f)$ and let $N$ denote the corresponding locally trivial subfactor of $M, N=\{x \oplus \theta(x) \mid x \in f M f\}$. If $N_{0}$ is a subfactor of $M$ which forms a commuting square with $N \subset M$, i.e. $E_{N_{0}} E_{N}=E_{N_{0} \cap N}$, and if $N_{0} \not \subset N$ then $f \in\left(N_{0} \cap N\right)^{\prime} \cap N_{0}$, and $N_{0} \cap N=\{x \oplus \theta(x) \mid x \in$ $\left.f N_{0} f\right\}$.

Proof. Let $N_{1}=N_{0} \cap N$. We have $1<\left[N_{0}: N_{1}\right] \leq[M: N]$. If $\left[N_{0}: N_{1}\right] \geq 4$ and $t_{0} \leq 1 / 2$ is such that $t_{0}\left(1-t_{0}\right)=\left[N_{0}: N_{1}\right]^{-1}$ then by 5.2 there is a projection $f_{0} \in N_{0}$ such that $E_{N_{1}}\left(f_{0}\right)=t_{0} 1$. Since $\left[N_{0}: N_{1}\right]^{-1} \geq[M: N]^{-1}$ it follows that $t_{0} \geq t$. By 3.2 it follows that $f_{0}=f, t_{0}=t$ and $[M: N]=\left[N_{0}: N_{1}\right]$. Thus $f \in N_{0}$ and since $f \in N^{\prime}$ we have $f \in N_{1}^{\prime} \cap N_{0}$. Moreover $\left[N_{0}: N_{1}\right]=\tau(f)^{-1}+\tau(1-f)^{-1}$ and Jones' formula [6] imposes $N_{1} f=f N_{0} f, N_{1}(1-f)=(1-f) N_{0}(1-f)$. Since $N_{1} \subset N$ we must then have $N_{1}=\left\{x \oplus \theta(x) \mid x \in f N_{0} f\right\}$.

Now if $\left[N_{0}: N_{1}\right]<4$ then $N_{1}^{\prime} \cap N_{0}=\mathbf{C}$ by [6]. Thus if $f_{0} \in N_{0}$ is a projection so that $E_{N_{1}}\left(f_{0}\right)=\left[N_{0}: N_{1}\right]^{-1} 1$, then

$$
E_{N}\left(f_{0}\right)=\left[N_{0}: N_{1}\right]^{-1} 1_{M}
$$

and since $E_{N_{1}^{\prime} \cap N_{0}}\left(f_{0}\right)=\tau\left(f_{0}\right) 1, E_{N^{\prime} \cap M}\left(f_{0}\right)$ will also be a scalar multiple of the identity. Since $\alpha=\tau\left(f_{0}\right)>1 / 4$ it follows by 5.1 that $\alpha=P_{k-1}\left([M: N]^{-1}\right) /[M: N] P_{k}\left([M: N]^{-1}\right)$ for some $k \geq 0$. It turns out that for projections $f_{0}$ in $M$ which expected on the locally trivial subfactor $N$ are scalars we must have $E_{N^{\prime} \cap M}\left(f_{0}\right)=a f+b(1-f)$, with $a \neq b$, so that $E_{N^{\prime} \cap M}\left(f_{0}\right)$ can never be a scalar. This contradiction will end the proof of the theorem. The actual computation of $a$ and $b$ is contained in the next:

6.3. Lemma. Let $N \subset M$ be a locally trivial subfactor like in 6.2 and $\lambda=[M: N]^{-1}=t(1-t)$. If $f_{0} \in M$ is such that $E_{N}\left(f_{0}\right)=$ $\alpha 1_{N}$ where $\alpha=\lambda P_{k-1}(\lambda) / P_{k}(\lambda)$, for some $k \geq 0$, then $E_{N^{\prime} \cap M}\left(f_{0}\right)=$ $a_{k} f+b_{k}(1-f)$ with $a_{k}, b_{k}$ satisfying the recursive relations: $a_{k}=$ $(1-t)^{2}\left(1-b_{k-1}\right)\left(P_{k}(\lambda) / P_{k+1}(\lambda)\right)^{2}, a_{k} t+b_{k}(1-t)=\lambda P_{k-1}(\lambda) / P_{k}(\lambda)$. 
The sequence $\left\{a_{k}\right\}_{k \geq 0}$ is increasing from $(1-t)^{2}$ to 1 and the sequence $\left\{b_{k}\right\}_{k \geq 0}$ is decreasing from $t^{2}$ to 0 . Moreover $a_{k}>b_{k}$ for all $k \geq 0$.

Proof of 6.3. Let first $f_{0}=e_{0}$ be the projection in $M$ with $E_{N}\left(f_{0}\right)=$ [M:N] $]^{-1} 1_{N}=t(1-t) 1_{N}=\lambda 1_{N}$. By the first part of the proof of 3.2 we have $\tau\left(e_{0} f\right)=(1-t) \tau\left(e_{0}\right)$. On the other hand we have $\tau\left(e_{0} f\right)=\tau\left(E_{N^{\prime} \cap M}\left(e_{0} f\right)\right)=\tau\left(E_{N^{\prime} \cap M}\left(e_{0}\right) f\right)=\tau\left(\left(a_{0} f+b_{0}(1-f)\right) f\right)=$ $a_{0} \tau(f)=a_{0} t$. Thus $a_{0} t=t(1-t)^{2}$ so that $a_{0}=(1-t)^{2}$ and $b_{0}=$ $\left(\tau\left(e_{0}\right)-a_{0} t\right) / \tau(1-f)=t^{2}$.

Let further $M_{-2} \subset N=M_{-1}$ be a subfactor of $N$ so that $M$ be the extension of $M_{-1}=N$ by $M_{-2}$ and so that $\left[e_{0}, M_{-2}\right]=0$. By [12] $M_{-2}$ is a locally trivial subfactor of $M_{-1}=N$. Let $f_{1} \in$ $M_{-1}$ be a projection so that $E_{M_{-2}}\left(f_{1}\right)=\lambda P_{k-1}(\lambda) / P_{k}(\lambda)$. Then $p_{0}=$ $P_{k}(\lambda) / P_{k+1}(\lambda)\left(1-f_{1}\right) e_{0}\left(1-f_{1}\right)$ is a projection in $\left(1-f_{1}\right) M\left(1-f_{1}\right)$ and $E_{\left(1-f_{1}\right) N\left(1-f_{1}\right)}\left(p_{0}\right)=\lambda P_{k}(\lambda) / P_{k+1}(\lambda)$ (by 4.1). Let $f^{\prime} \in M_{-2}^{\prime} \cap M_{-1}$ be the projection with $\tau\left(f^{\prime}\right)=t$. Since $f e_{0} f=(1-t)\left(1-f^{\prime}\right) f$ we then have

$$
\begin{aligned}
& \tau\left(1-f_{1}\right) \tau_{1-f_{1}}\left(p_{0} f\right)=\frac{P_{k}(\lambda)}{P_{k+1}(\lambda)} \tau\left(\left(1-f_{1}\right) e_{0}\left(1-f_{1}\right) f\right) \\
& \quad=\frac{P_{k}(\lambda)}{P_{k+1}(\lambda)} \tau\left(\left(1-f_{1}\right) e_{0} f\right)=\frac{P_{k}(\lambda)}{P_{k+1}(\lambda)} \tau\left(E_{M_{-2}^{\prime} \cap M}\left(1-f_{1}\right) e_{0} f\right) \\
& \quad=\frac{P_{k}(\lambda)}{P_{k+1}(\lambda)}(1-t) \tau\left(E_{M_{-2}^{\prime} \cap M}\left(1-f_{1}\right)\left(1-f^{\prime}\right) f\right) .
\end{aligned}
$$

But by induction we have

$$
E_{M_{-2}^{\prime} \cap M}\left(1-f_{1}\right)=\left(1-a_{k-1}\right) f^{\prime}+\left(1-b_{k-1}\right)\left(1-f^{\prime}\right) .
$$

Thus

$$
\frac{P_{k+1}(\lambda)}{P_{k}(\lambda)} \tau_{1-f_{1}}\left(p_{0} f\right)=\frac{(1-t)^{2}}{\tau\left(1-f_{1}\right)} t\left(1-b_{k-1}\right) .
$$

But we also have $\tau_{1-f_{1}}\left(p_{0} f\right)=a_{k} t$. It follows that

$$
\begin{aligned}
a_{k} & =\frac{P_{k}(\lambda)}{P_{k+1}(\lambda)} \tau\left(1-f_{1}\right)^{-1}(1-t)^{2}\left(1-b_{k-1}\right) \\
& =\left(\frac{P_{k}(\lambda)}{P_{k+1}(\lambda)}\right)^{2}(1-t)^{2}\left(1-b_{k-1}\right) .
\end{aligned}
$$

That $a_{k}$ is increasing and $b_{k}$ is decreasing follows now by induction and by 2.9. The limits $a$ and $b$ will then satisfy $a=\lim a_{k}=$ $\lim \left(P_{k}(\lambda) / P_{k+1}(\lambda)(1-t)\right)^{2}\left(1-\lim b_{k-1}\right)=1-b$ and $a t+b(1-t)=t$. But this implies $a=1, b=0$. Moreover, since $a_{0}>b_{0}$ and since $a_{n}$ is increasing and $b_{n}$ is decreasing, it follows that $a_{n}>b_{n}$ for all $n$. 
This shows in particular that $E_{N^{\prime} \cap M}\left(f_{0}\right) \neq \tau\left(f_{0}\right) 1$ for any projection which expected on $N$ gives a scalar. This completes the proof of the lemma and thus also of the theorem.

6.4. Remark. As we mentioned in 4.6 the values $\lambda P_{k-1}(\lambda) / P_{k}(\lambda)$ of the traces of projections that expect on scalars coincide with the values of the Markov traces on the projections coming from representations of the generators of the Hecke algebras. There naturally arises the question whether we can choose projections $f_{0} \in M_{0}, f_{1} \in$ $M_{1}, f_{2} \in M_{2}, \ldots$, in the Jones' tower of factors associated to the inclusion $N \subset M$, so that $E_{M_{i-1}}\left(f_{i}\right)=\lambda P_{k-1}(\lambda) / P_{k}(\lambda)$ for all $n \geq i \geq 0$ and so that $\left\{f_{i}\right\}_{n \geq i \geq 0}$ generate the semisimple Hecke algebra corresponding to the parameter $\lambda$ as in [17]. This is in fact impossible. Indeed if this would be true we could get a contradiction by using Theorem 5.1. Another way to get the contradiction is by using [12] and one of Wenzl's results in [17]: if $A_{i}=\operatorname{Alg}\left\{1, f_{0}, \ldots, f_{i-1}\right\}, B_{i}=\operatorname{Alg}\left\{1, f_{0}, \ldots, f_{i}\right\}$, where $f_{i}$ are as before and satisfy the Hecke algebra axioms $f_{i} f_{i+1} f_{i}-$ $\lambda f_{i}=f_{i+1} f_{i} f_{i+1}-\lambda f_{i+1}$, then $E_{B_{i}} E_{M_{t-1}}=E_{M_{i-1}} E_{B_{i}}=E_{A_{i}}$ and by [12] $\lim _{i} \lambda\left(B_{i}, A_{i}\right)$ coincides with the inverse of the index of the corresponding pair of subfactors (associated with the corresponding representations of Hecke algebras in [17]). By [17] this index is equal to $\lambda^{-k-1} P_{k}(\lambda)^{2}$ so it is strictly larger than $\lambda^{-1}$. But since $A_{i} \subset B_{i}$ and $M_{i-1} \subset M_{i}$ form a commuting square, $\lambda=\lambda\left(M_{i}, M_{i-1}\right) \leq \lambda\left(B_{i}, A_{i}\right)$, which is impossible if $i$ is large enough.

7. A related dilation problem. Instead of a subfactor of finite index $N \subset M$ we may of course consider arbitrary von Neumann subalgebras $B \subset M$ and let $\Lambda(M, B)$ be defined like in 1.1 to be the invariant up to conjugacy by automorphisms of $M$. There are at least two special cases that seem of most interest, when $B$ is a subfactor and when $B$ is a maximal abelian subalgebra of $M$.

7.1. Proposition. If $B$ is either a subfactor of infinite index and trivial relative commutant of $M$ or an abelian subalgebra of $M$ then $\overline{\Lambda(M, B)}=[0,1]$. If $B$ is maximal abelian and the normalizer of $B$ in $M$ generates a type $\mathrm{II}_{1}$ von Neumann subalgebra of $M$ then $\Lambda(M, B)=$ $[0,1]$.

Proof. If $B$ is abelian then let $\tilde{B} \subset M$ be a maximal abelian von Neumann subalgebra of $M$ containing $B$. If $\alpha \in \Lambda(M, \tilde{B})($ or $\overline{\Lambda(M, \tilde{B}})$ ) then clearly $\alpha \in \Lambda(M, B)$ (respectively $\overline{\Lambda(M, B})$ ). But arguing as in 
[16] it is easy to see that any rational number lies in $\Lambda(M, \tilde{B})$. If $B \subset$ $M$ is so that $B^{\prime} \cap M=\mathrm{C}$ and $[M: B]=\infty$ then a similar proof as that of 2.3 in [12] shows that $\overline{\Lambda(M, B)}=[0,1]$. If $B$ is maximal abelian and $N=\mathscr{N}(B)^{\prime \prime}$ is a type $\mathrm{II}_{1}$ von Neumann subalgebra then there exists $B_{0} \subset B$ and $R \subset N$ a hyperfinite subfactor generated by unitaries normalizing $B_{0}$ so that $B_{0}$ is maximal abelian in $R$ (see e.g. [16]). But then by Connes-Feldman-Weiss theorem [3] we may assume there exist unitary elements $u \in B_{0}, v \in R$ so that $u$ generates $B_{0}$, and $v u=e^{2 \pi i t} u v$ for some irrational number $t \in[0,1]$. Then any projection in the von Neumann algebra generated by $v$ projects on $B_{0}$ on a scalar. Since such projections may have any trace, this shows that $\Lambda\left(R, B_{0}\right)=[0,1]$. But it is easy to see by construction that $E_{R} E_{B}=B_{B_{0}}$. Thus $\Lambda(M, B) \supset \Lambda(R, B) \supset \Lambda\left(R, B_{0}\right)=[0,1]$.

There is also another interesting possible problem: we may consider instead of the set $\Lambda(M, B)$ the set of all possible relative dimensions $\tilde{\Lambda}(M, B)=\left\{E_{B}(e) \mid e\right.$ projection in $\left.M\right\}$ or the set $\tilde{\Lambda}_{0}(M, B)=\{f$ : $[0,1] \rightarrow[0,1] \mid f(t)$ is the trace of the spectral projection of $E_{B}(e)$ corresponding to the interval $[t, \infty]\}$. It would be interesting to compute these sets at least in the most simple examples (e.g. $B$ semiregular). It is not difficult to show that if $B$ is maximal abelian then $\bar{\Lambda}(M, B)$ coincides with the unit ball of the positive cone of $B$.

The problem of finding whether an element $a \in B, 0 \leq a \leq 1$ is the image by $E_{B}$ of a projection $e \in M$ can alternately be regarded as a dilation problem. Indeed if $a$ is given then there exists a unique unital completely positive map $\varphi: \mathbf{C}^{2} \rightarrow B$ so that $\varphi((1,0))=a, \varphi((0,1))=$ $1-a$. To show that there exists a projection $e \in M$ so that $E_{B}(e)=a$ is the same as to show that there is a *-morphism $\pi: \mathbf{C}^{2} \rightarrow M$ so that the compression of $\pi$ to $L^{2}\left(B, \tau_{\mid B}\right)$ coincides with $\varphi$ (where $B$ is viewed in its representation on $\left.L^{2}(B, \tau)\right)$. Instead of $C^{2}$ we may of course consider other algebras:

7.2. Problem. Study special classes of completely positive maps $\varphi: A_{0} \rightarrow B$ which can be dilated to $M$, i.e., for which there exists a *-morphism $\pi: A_{0} \rightarrow M$ so that $\varphi=E_{B} \pi$.

In the case $N \subset M$ is a subfactor of finite index then the small values in $\tilde{\Lambda}, \tilde{\Lambda}_{0}$ can be completely characterized, in particular also the dilation problem for $A_{0}=\mathrm{C}^{2}$. More precisely we have:

7.3. Proposition. Suppose $e_{0} \in M$ is so that $E_{N}\left(e_{0}\right)=\lambda 1_{N}$ where $\lambda=[M: N]^{-1}>0$. Let $N_{1} \subset N$ be so that $M, e_{0}$ come from the 
basic construction for the inclusion $N_{1} \subset N$ (i.e., $N_{1}=N \cap\left\{e_{0}\right\}^{\prime}$, $\left.\left[N: N_{1}\right]=[M: N]\right)$. If $e \in M$ is a projection so that $E_{N}(e) \leq \lambda 1_{N}$ then there exist a projection $f \in N_{1}$ and a unitary element $w \in N$ so that $e=w e_{0} f w^{*}$ and so that $E_{N}(e)=\lambda w f w^{*}$. In particular, if $e^{\prime}, e^{\prime \prime} \in M$ are projections of the same trace and $E_{N}\left(e^{\prime}\right) \leq \lambda 1, E_{N}\left(e^{\prime \prime}\right) \leq \lambda 1$ then $e^{\prime}, e^{\prime \prime}$ are conjugate by a unitary element in $N$.

Proof. By hypothesis and 2.1 in [12] we have $\lambda e \geq e E_{N}(e) e \geq \lambda e$. But this implies that $(1-e) E_{N}(e) e=0$, hence $\left[E_{N}(e), e\right]=0$. It follows that if the spectrum of $E_{N}(e)$ would contain a value distinct of 0 and $\lambda$ then the projection $f^{\prime}$ defined to be the spectral projection of $E_{N}(e)$ corresponding to $(0, \alpha]$ would satisfy $\left[f^{\prime}, e\right]=0,0 \neq E_{N}\left(f^{\prime} e\right)=$ $E_{N}(e) f^{\prime} \leq \alpha 1<\lambda 1$, a contradiction. Thus $E_{N}(e)=\lambda f^{\prime \prime}$ for some projection $f^{\prime \prime} \in N$. Since $f^{\prime \prime} \geq E_{N}(e), f^{\prime \prime} \geq e$. Since $N$ is a factor, there exists a unitary element $w_{0} \in N$ so that $f=w_{0}^{*} f^{\prime \prime} w_{0} \in N_{1}=N^{\prime} \cap\left\{e_{0}\right\}$. Then in $M_{f}$ we have the projections $e_{0} f$ and $w_{0}^{*} e w_{0}$, and $E_{N_{f}}\left(e_{0} f\right)=$ $E_{N_{f}}\left(w_{0}^{*} e w_{0}\right)=\lambda f=\lambda 1_{N_{f}}$. By [12] there exists a unitary element $w_{1} \in N_{f}$ so that $w_{1}^{*} w_{0}^{*} e w_{0} w_{1}=e_{0} f$. Thus $f$ and $w=w_{0}\left(w_{1}+(1-f)\right)$ satisfy the requirements.

Added in proof. After this work had been completed we learned that the inclusion $\Lambda(M, N) \subset\left\{\alpha \geq 0 \mid \exists f \in M\right.$ such that $\left.E_{N}(f)=\alpha 1\right\}$, which is part of the equation in Theorem 5.1, as obtained independently by A. Ocneanu.

\section{REFERENCES}

[1] A. Connes, Classification of injective factors, Ann. of Math., 104 (1976), 73-115.

[2] Classification des facteurs, Proc. Symp. Pure Math., 38 (1982), part 2, 43-109.

[3] A. Connes, J. Feldman and B. Weiss, An amenable equivalence relation is generated by a single transformation, Ergodic Theory and Dynamical Systems, 1 (1981), 431-450.

[4] H. Dye, On groups of measure preserving transformations II, Amer. J. Math., 85 (1963), 551-576.

[5] F. Goodman, P. de la Harpe and V.F.R. Jones, Coxeter graphs and towers of algebras, to appear.

[6] V.F.R. Jones, Index for subfactors, Invent. Math., 72 (1983), 1-25.

[7] Hecke algebra representations of braid groups and link polynomials, Ann. Math., 126 (1987), 335-388.

[8] - Subfactors and related topics, Proc. of the U.S. - W.K Seminar in operator algebras, Warwick 1987.

[9] F. Murray and J. von Neumann, On rings of generators IV, Ann. Math., 44 (1943), 716-808. 
[10] A. Ocneanu, P. Freyd, D. Yetter, J. Hoste, W. Likorish and K. Millet, A new polynomial invariant of knots and links, Bull. Amer. Math. Soc., 12 (1985), 183-189.

[11] A. Ocneanu, in preparation.

[12] M. Pimsner and S. Popa, Entropy and index for subfactors, Ann. Sci. Ec. Norm. Sup., 19 (1986), 57-106.

[13] Finite dimensional approximation of pairs of algebras and obstructions for the index, preprint 1988.

[14] S. Popa, Correspondences, preprint 1986.

[15] Maximal injective subalgebras in factors associated with free groups, Adv. in Math., 50 (1983), 27-48.

[16] On a problem of $R$. V. Kadison on maximal abelian *-subalgebras in factors, Invent. Math., 65 (1981), 269-281.

[17] H. Wenzl, Hecke algebras of type $A_{n}$ and subfactors, Invent. Math., 92 (1988), 349-383.

[18] - On sequences of projections, C.R. Math. Rep. Acad. Sci. Canada, 9 (1987), 5-9.

Received August 29, 1988.

UNIVERSITY OF CALIForNiA

Los ANGeles, CA 90024-1555-05 



\section{PACIFIC JOURNAL OF MATHEMATICS EDITORS}

\author{
V. S. VARADARAJAN \\ (Managing Editor) \\ University of California \\ Los Angeles, CA 90024 \\ Herbert Clemens \\ University of Utah \\ Salt Lake City, UT 84112 \\ THOMAS ENRIGHT \\ University of California, San Diego \\ La Jolla, CA 92093
}

R. FINN

Stanford University

Stanford, CA 94305

HeRmanN FlaschKa

University of Arizona

Tucson, AZ 85721

VAUGHAN F. R. JONES

University of California

Berkeley, CA 94720

STEVEN KeRCKHOFF

Stanford University

Stanford, CA 94305
RoBION KIRBY

University of California

Berkeley, CA 94720

C. C. MOore

University of California

Berkeley, CA 94720

HAROLD STARK

University of California, San La Jolla, CA 92093

\section{ASSOCIATE EDITORS}
R. ARENS
E. F. BECKENBACH
B. H. NeUmanN
F. WolF
K. YosHIDA (1906-1982)

\section{SUPPORTING INSTITUTIONS}
UNIVERSITY OF ARIZONA
UNIVERSITY OF BRITISH COLUMBIA CALIFORNIA INSTITUTE OF TECHNOLOGY UNIVERSITY OF CALIFORNIA MONTANA STATE UNIVERSITY UNIVERSITY OF NEVADA, RENO NEW MEXICO STATE UNIVERSITY OREGON STATE UNIVERSITY
UNIVERSITY OF OREGON UNIVERSITY OF SOUTHERN CALIFORNIA STANFORD UNIVERSITY UNIVERSITY OF HAWAII UNIVERSITY OF TOKYO UNIVERSITY OF UTAH WASHINGTON STATE UNIVERSITY UNIVERSITY OF WASHINGTON 


\section{Pacific Journal of Mathematics}

\section{Vol. 137, No. $1 \quad$ January, 1989}

V. S. Varadarajan, Henry Abel Dye $\ldots \ldots \ldots \ldots \ldots \ldots \ldots \ldots \ldots \ldots$ iii

Huzihiro Araki, An application of Dye's theorem on projection lattices to orthogonally decomposable isomorphisms $\ldots \ldots \ldots \ldots \ldots \ldots \ldots \ldots \ldots$

Richard Arens, The limit of a sequence of squares in an algebra need not be

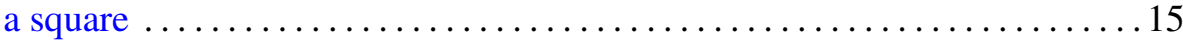

William Arveson, An addition formula for the index of semigroups of

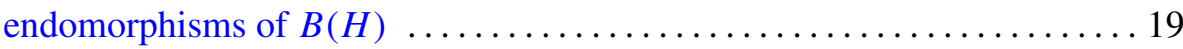

Robert James Blattner and Susan Montgomery, Crossed products and

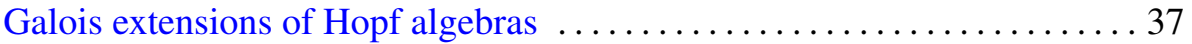

Erik Christensen and Allan M. Sinclair, On the vanishing of $H^{n}\left(\mathscr{A}, \mathscr{A}^{*}\right)$

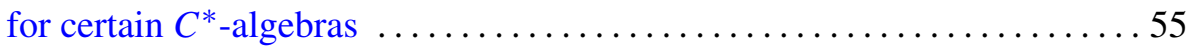

Philip C. Curtis, Jr. and Michael M. Neumann, Nonanalytic functional

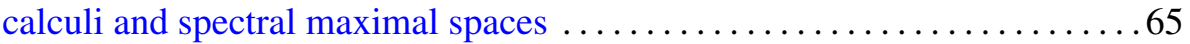

George A. Elliott and David E. Handelman, Addition of $C^{*}$-algebra extensions

Yaakov Friedman and Bernard Russo, Some affine geometric aspects of

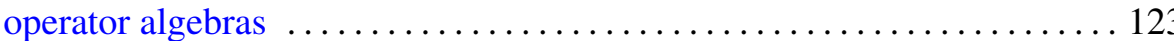

Valentin Ya. Golodets and Sergey D. Sinelshchikov, Regularization of actions of groups and groupoids on measured equivalence relations . . . 145

Irving Kaplansky, CCR-rings 155

Hideki Kosaki, Characterization of crossed product (properly infinite case)

Gert Kjærgaard Pedersen, Three quavers on unitary elements in $C^{*}$-algebras

Sorin Popa, Relative dimension, towers of projections and commuting squares of subfactors

Martin E. Walter, On a new method for defining the norm of

Fourier-Stieltjes algebras 